\title{
Early Detection of Baby-Rain-Cell Aloft in a Severe Storm and Risk Projection for Urban Flash Flood
}

\author{
Eiichi Nakakita, ${ }^{1}$ Hiroto Sato, ${ }^{2}$ Ryuta Nishiwaki, ${ }^{3}$ \\ Hiroyuki Yamabe, ${ }^{4}$ and Kosei Yamaguchi ${ }^{1}$ \\ ${ }^{1}$ Disaster Prevention Research Institute, Kyoto University, Uji, Kyoto 611-0011, Japan
${ }^{2}$ Graduate School of Engineering, Kyoto University, Uji, Kyoto 611-0011, Japan
${ }^{3}$ Sumitomo Heavy Industries, Ltd., Shinagawa, Tokyo 141-6025, Japan
${ }^{4}$ Tokyo Electric Power Company Holdings, Inc., Chiyoda, Tokyo 100-8560, Japan
}

Correspondence should be addressed to Eiichi Nakakita; nakakita@hmd.dpri.kyoto-u.ac.jp

Received 22 July 2016; Revised 10 November 2016; Accepted 15 December 2016; Published 22 January 2017

Academic Editor: Ke Zhang

Copyright (C) 2017 Eiichi Nakakita et al. This is an open access article distributed under the Creative Commons Attribution License, which permits unrestricted use, distribution, and reproduction in any medium, provided the original work is properly cited.

\begin{abstract}
In July 2008, five people were killed by a tragic flash flood caused by a local torrential heavy rainfall in a short time in Toga River. From this tragic accident, we realized that a system which can detect hazardous rain-cells in the earlier stage is strongly needed and would provide an additional 5 to $10 \mathrm{~min}$ for evacuation. By analyzing this event, we verified that a first radar echo aloft, by volume scan observation, is a practical and important sign for early warning of flash flood, and we named a first echo as a "baby-rain-cell" of Guerrilla-heavy rainfall. Also, we found a vertical vorticity criterion for identifying hazardous rain-cells and developed a heavy rainfall prediction system that has the important feature of not missing any hazardous rain-cell. Being able to detect heavy rainfall by $23.6 \mathrm{~min}$ on average before it reaches the ground, this system is implemented in XRAIN in the Kinki area. Additionally, to resolve the relationship between baby-rain-cell growth and vorticity behavior, we carried out an analysis of vorticity inside baby-rain-cells and verified that a pair of positive and negative vertical vortex tubes as well as an updraft between them existed in a rain-cell in the early stage.
\end{abstract}

\section{Introduction}

On July 28, 2008, a tragic flash flood occurred in Toga River, Kobe City, Japan, washing away fifty people who were enjoying sunny weather and playing in river parks; this disaster resulted in five fatalities. This disaster drew much attention from government authorities and the engineering community for investigation of disaster factors. A survey report [1] showed that the runoff response of this incident was about $10 \mathrm{~min}$, and most of the flood water came from the sewer system of urban areas during a severely localized heavy rainfall. Therefore, this tragic disaster was the result of a combination of paved urban area and severely localized heavy rainfall.

Recently, weather analyses have shown that localized heavy rainfalls have become increasingly frequent during the summer season. In Japan, there are many short rivers similar to Toga River with small basins about only $10 \mathrm{~km}^{2}$ and sewage systems, and most of the rivers are used as public parks. Thus, these public parks in rivers are likely to be dangerous during severely localized heavy rainfall events, for example, $30 \mathrm{~min}$ rainfall at an intensity of $50 \mathrm{~mm} / \mathrm{h}$.

In addition, based on photos recorded by a public monitoring camera near the location of the flood incident, there was no obvious in situ sign before the sudden approach of the flood. This indicates that the time for response after the flood arrived was not sufficient. Therefore, it is imperative to develop a methodology for early warning of flash floods before localized heavy rainfalls reach the ground. Such localized torrential rainfalls are known as "Guerrilla-heavy rainfalls," which is a popular term in Japanese news media denoting a short localized heavy rainfall. A Guerrilla-heavy rainfall is generally formed by even an isolated cumulonimbus cloud which is suddenly generated aloft and develops rapidly in a very short time. Therefore, it is very difficult to predict a flash flood caused by Guerrilla-heavy rainfall. For 
example, in the Toga River case, there was only about ten minutes for the operating radar, which observed using Plan Position Indicator (PPI) scans at a low elevation angle, to receive signals of the rainfall event before the flood reached the incident location. Thus, in order to prevent such flash flood disasters, it is very necessary to develop a system that can detect hazardous rain-cells in the earlier stage to save more than 5 to $10 \mathrm{~min}$ and issue an alert for people to evacuate from river parks to a higher position before flood arrival. This is the main objective of the present research.

In this paper, we shall propose a novel Guerrilla-heavy rainfall prediction system. The structure of this paper is as follows. Section 2 introduces an analysis of the Toga River event using a C-band radar. The result shows that monitoring by volume scan operation is very effective for observing a first radar echo of a rain-cell in an upper atmospheric layer and facilitates detection of a severe storm that might cause Guerrilla-heavy rainfall. Further, Section 3 describes a method for identifying hazardous rain-cells that may cause Guerrilla-heavy rainfalls using vertical vorticity values inside rain-cells obtained by Doppler radar observation. Then, the novel Guerrilla-heavy rainfall prediction system is introduced. To resolve the relationship between growth of a rain-cell and vorticity inside the rain-cell, Section 4 presents an analysis of the structure of vertical vortex tubes inside rain-cells. Finally, Section 5 summarizes and discusses the advantages of the system as well as our ongoing research.

\section{Early Detection of Rain-Cells Using Volume Scan Radar Observation}

In this study, early detection is defined as detecting the existence of a first radar echo from a rain-cell in an upper atmospheric layer. This is because it is very essential to broadcast the risk information of a flash flood to the public people before a raindrop reaches the ground or before a tragic flash flood approaches even if the information is distributed at the timing of first radar echo appearance aloft.

Nakakita et al. [2] analyzed the Toga River flash flood event and verified that the first radar echo in an upper atmospheric layer was detected through a volume scan observation with an operational C-band radar. Nakakita et al. [3] named a first radar echo aloft in a cumulonimbus cloud as a "baby-rain-cell" and emphasized that a baby-raincell was a very effective sign for issuing early flash flood evacuation warnings. Based on this research, the Ministry of Land, Infrastructure, Transport and Tourism (MLIT) of Japan has finished installing a new weather radar network, called "X-band polarimetric RAdar Information Network" (XRAIN), in major urban areas around Japan since 2010 in order to prevent flash flood disasters through faster detection of localized heavy rainfall events.

In the following section, we present an analysis of the Toga River event using a C-band radar as well as the introduction of XRAIN.

2.1. Analysis of Toga River Event Using a C-Band Radar. Toga River, which is located in Kobe City in Japan, is a popular place for people to enjoy the waterfront during sunny days in the summer season (see Figure 1(a) for field photo). On July 28,2008 , as usual, many people were enjoying the sunny weather in the river parks in Toga River. A slight shower occurred, and then, without any significant sign or time of react, a flash flood surge suddenly struck the river parks at 14:42 and washed away the people. Figure 1(b) shows field snapshots at different times of the day in Toga River during this flood event. As is shown in Figure 2, according to figures of rainfall intensity from low angle observation by C-band radars operated by the MLIT, heavy rainfall occurred over the basin of Toga River at 14:35. This means that seven minutes was available to issue a flash flood warning. Seven minutes may appear to be sufficient for issuing an evacuation warning; however, in fact, it took about ten minutes to finalize procedures of making figures and distributing it to the public officials. Thus, conventional methods for rainfall observation may not provide early enough detection to protect people from disasters caused by flash floods. Therefore, it is emphasized that an additional five to ten minutes for early detection of heavy rainfall is absolutely and imperatively required.

In the last few decades, for scientific purposes, many researchers have focused on a first radar echo of a thunderstorm to analyze cloud structure, for example, [4, 5], and to understand the mechanism of precipitation onsets, for example, $[6,7]$. We focused on a first radar echo and attempted to use it as a sign of early detection [2]. It should be noted that while there have been many studies focused on a first radar echoes, this was the first trial utilizing a first radar echo in a practical rainstorm prediction algorithm based on an operational radar network.

We analyzed the three-dimensional structure of reflectivity in the upper atmospheric layer over the target basin using volume scan radar observation data, as is shown in Figure 3 [2]. Data were obtained from the Miyama C-band radar, operated by the MLIT and the time interval of observation was seven minutes and thirty seconds. The first echo of the hazardous cloud in the upper atmospheric layer was captured at around 14:13. At that time, any raindrops have not reached the ground yet. Most importantly, data from volume scan observations indicate that a hazardous cloud was detected about $30 \mathrm{~min}$ before the flood occurrence. According to this result, it is obvious that detecting a first radar echo is quite effective to predict a flash flood. Hence, we termed a first radar echo aloft in a cumulonimbus cloud as a "baby-raincell" and argued that a baby-rain-cell is a very effective sign for issuing an early flood evacuation warning [3].

To sum up, the three-dimensional information provided by volume scan mode has been proven to be practical and useful for the detection of hazardous rain-cells in the early stage.

2.2. New Japanese Radar Network Using X-Band Radars. Owing to the advantage of volume scan observations [2], the MLIT has started installing many X-band radars, which provide volume scan observation data with high spatial and temporal resolutions, throughout Japan. Table 1 lists the specifications of the installed X-band radar and the Miyama $\mathrm{C}$-band radar in 2008. We can see the X-band radar has much 


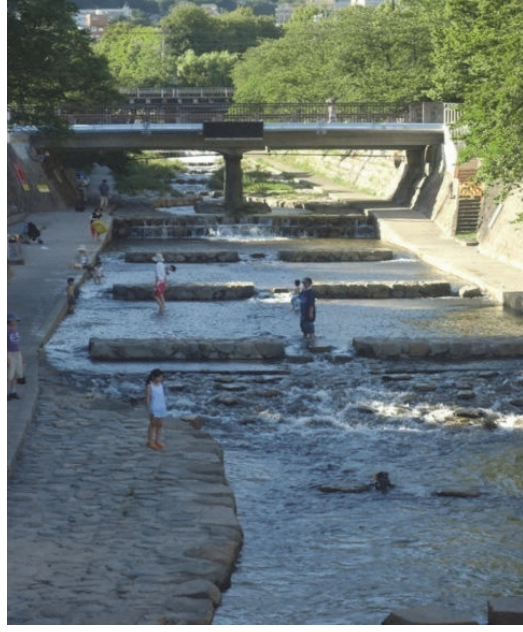

(a)
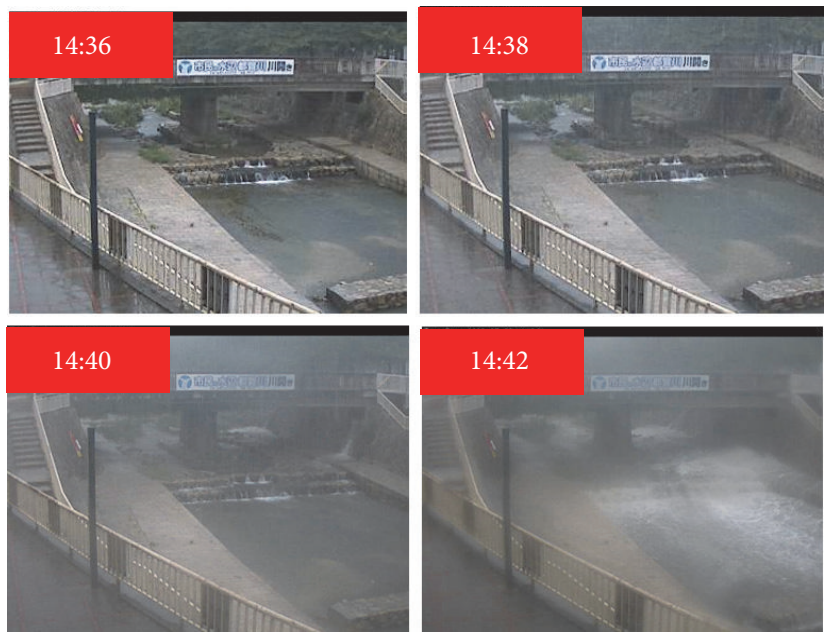

(b)

Figure 1: (a) Photograph of Toga River (July 17, 2012). (b) Field snapshots at different times in Toga River during the flood event.

TABLE 1: List of specifications of the installed X-band radars and the Miyama C-band radar.

\begin{tabular}{lcc}
\hline Radar name & $\begin{array}{c}\text { X-band } \\
\text { multiparameter radar } \\
\text { Time resolution }\end{array}$ & $\begin{array}{c}\text { Miyama C-band } \\
\text { radar in 2008 }\end{array}$ \\
$\begin{array}{l}\text { Range resolution } \\
\text { (maximum range) }\end{array}$ & $150 \mathrm{~m}(80 \mathrm{~km})$ & $300 \mathrm{~m}(120 \mathrm{~km})$ \\
Azimuth resolution & $1.2^{\circ}$ & $1.4^{\circ}$ \\
Parameters & $\begin{array}{c}\text { Reflectivity, Doppler } \\
\text { velocity, polarimetric } \\
\text { parameters }\end{array}$ & $\begin{array}{c}\text { Reflectivity, Doppler } \\
\text { velocity }\end{array}$ \\
\hline
\end{tabular}

higher spatial and temporal resolutions than the Miyama Cband radar. Additionally, the X-band radar observes not only reflectivity and Doppler velocity but also various polarimetric parameters. These new parameters are used operationally for calculating the rainfall intensity as well as experimentally for many fundamental researches. However, compared with the C-band radar, the X-band radar has two disadvantages: (1) high rain attenuation and (2) short observation range. To overcome the two drawbacks, many similar concepts of densely overlapped radar observation networks have been proposed, for example, [8-10]. Following this concept, the MLIT has constructed a new weather radar network, XRAIN in major urban areas in Japan since 2010. As of now, the MLIT has finished installing $39 \mathrm{X}$-band radars. Figure 4 illustrates the radar distribution as well as the observation ranges. For more precise observation in some specific areas, more radars were installed for greater overlapping of the observation ranges. Each X-band radar finishes one volume scan observation in $5 \mathrm{~min}$. Additionally, to overcome the attenuation problem, the MLIT corrects the observed reflectivity and differential reflectivity $\left(Z_{\mathrm{DR}}\right)$ using specific differential phase $\left(K_{\mathrm{DP}}\right)[11,12]$. After correction, only the surface data from all individual radar is operationally processed and condensed together into one composite surface rain data with high spatial $(250 \mathrm{~m}$ by $250 \mathrm{~m}$ ) and temporal resolutions (one minute interval) [12]. The data is operationally distributed to the public as a standard real-time product [13]. However, different from the surface rain data, three-dimensional volume scan data is not distributed to the public. For different objectives, we processed the volume scan data in different manners. For earlier detection, volume scan data of four radars in the Kinki area was merged into three-dimensional Cartesian coordinate system, as is mentioned in Section 3; for basic analysis, volume scan data of each four radars was utilized directly without any interpolation or composition, as is shown in Section 4.

Then, with the data from XRAIN, Nakakita et al. [14] proposed a prediction methodology for Guerrilla-heavy rainfall using vertical vorticity values inside a rain-cell. This methodology is elaborated in Section 3.

\section{Early Detection and Risk Prediction System for Guerrilla-Heavy Rainfall}

As mentioned in Section 2, XRAIN is applicable for detection of baby-rain-cells aloft in the very early stage. However, as not all rain-cells aloft develop into severe storms, identification of the danger level of the detected rain-cell is a critical issue for precise flood warning. Nakakita et al. [14] found that rain-cells that caused Guerrilla-heavy rainfalls had vertical vorticity values greater than or equal to $0.03 \mathrm{~s}^{-1}$ inside the rain-cells and proposed a methodology for the prediction of Guerilla-heavy rainfalls by utilizing vertical vorticity, as introduced in the following section.

3.1. Vorticity in the Early Stage of a Supercell. Referring to cloud dynamics [15-17], the characteristic behavior of supercell evolution in the early stage is vortex tube tilting. Figure 5 schematically illustrates the early stage of the development of a cumulonimbus cloud that will develop into a supercell. The vertical shear of horizontal wind generates a horizontally 


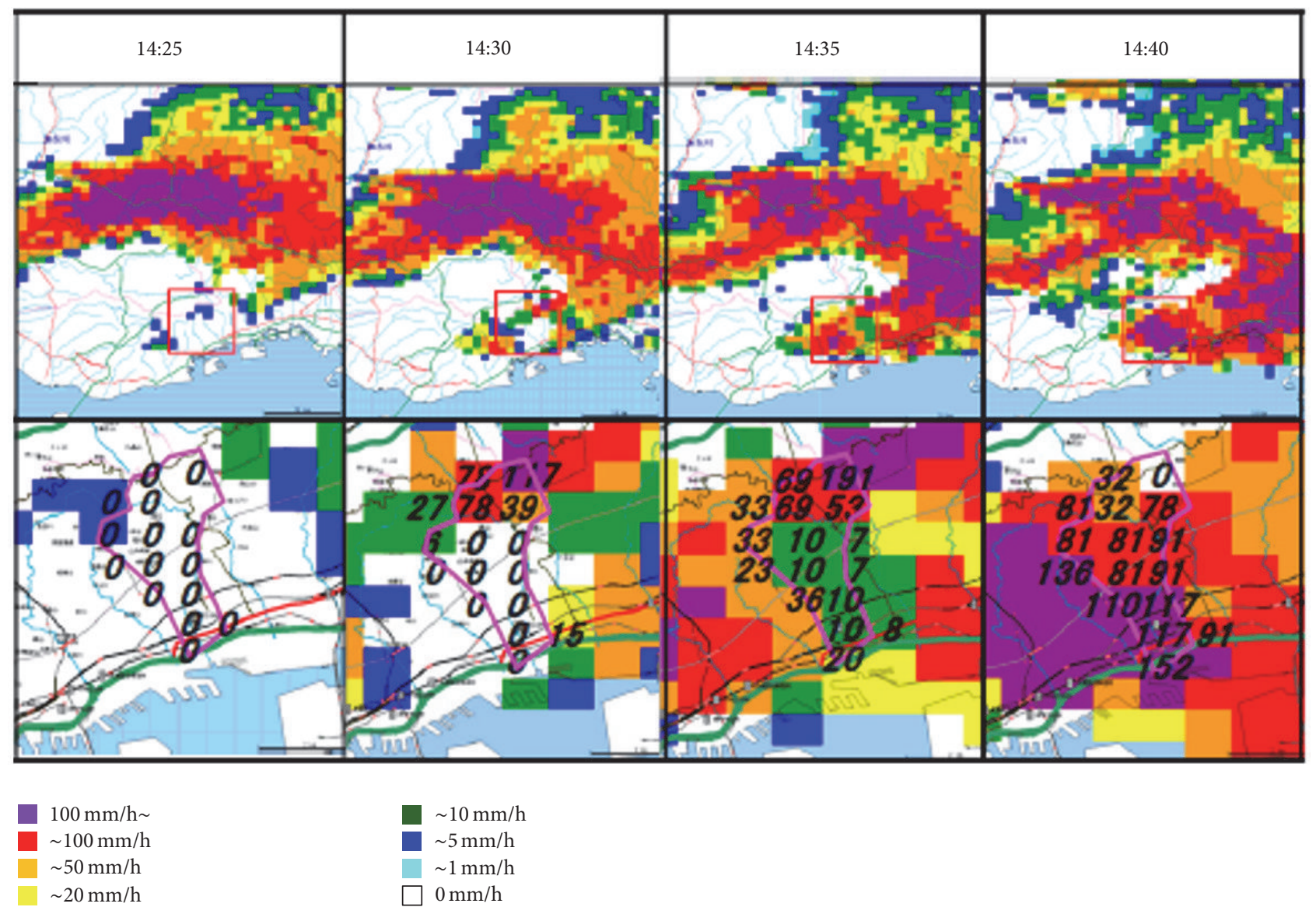

FIGURE 2: Rainfall intensity from low angle observation by C-band radars operated by the MLIT. Lower figures show zoom-in distributions in the range of the red rectangular in upper figures. The numbers in lower figures mean the radar estimated value of rainfall intensity.

oriented vortex tube, which is indicated by circular arrows in Figure 5(a). If this horizontal vortex tube meets an updraft, the tube is tilted and transferred into the vertical vortex tube along the updraft. Hence, a pair of positive and negative vertical vortex tubes appears in the rain-cell, as shown in Figure 5(b). However, even though the behavior of vortex tube tilting is well understood for the formation of a supercell in the early stage, the early stage of development of baby-raincells, which form Guerrilla-heavy rainfalls, is still unknown. Nevertheless, by physical intuition, a rain-cell for a Guerrillaheavy rainfall can be reasonably assumed to have the motion of vertical vorticities, analogous to a supercell. Therefore, in order to appropriately identify the potential danger level of a baby-rain-cell aloft, Nakakita et al. [18] proposed a methodology for identifying danger level using vertical vorticity values inside a baby-rain-cell. Data processing and efficacy verification of this method are described in the next section.

\subsection{Identification of Hazardous Rain-Cells Using Vertical Vorticity}

3.2.1. Methodology of Data Processing. Three-dimensional volume scan data from four radars in the Kinki area, as is shown in Figure 6, was used for the research. As one cycle for volume scan observation takes five minutes, the time interval for analysis was set to be five minutes. In this study, reflectivity and Doppler velocity in the polar coordinate system were used. For calculation of vorticity, all data were transformed into three-dimensional Cartesian coordinate system by interpolation. For the transformation, the initial dimension of a unit rectangular element for the two types of data is $200 \mathrm{~m} \times 200 \mathrm{~m} \times 200 \mathrm{~m}$. However, to exclude interruption from noisy echoes in the background, reflectivity data was further averaged into a larger unit element with a dimension of $500 \mathrm{~m} \times 500 \mathrm{~m} \times 250 \mathrm{~m}$, and its threshold was set to be $20 \mathrm{dBZ}$.

For calculation of vertical vorticity inside a baby-raincell, the transformed Doppler velocity data was used [18]. As Doppler velocity can only measure the radial component of a target's velocity relative to the radar, it cannot provide complete information of a target with a large tangential velocity component. This means that some estimations are required to obtain reasonable values of vorticity using Doppler velocity data. To solve this, we used Doppler velocity along the beam line with multiple radar observation. To calculate approximate vorticity, we introduced an eightdirection classification and eight different equations for more precise estimation [18], as shown in Figure 7. Then, at a given location, from all vorticities calculated using volume scan 

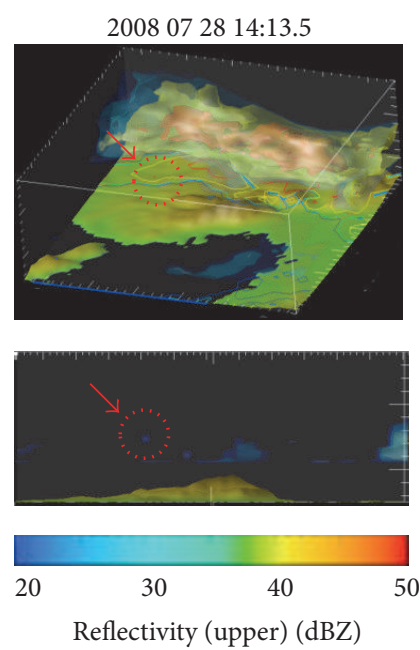
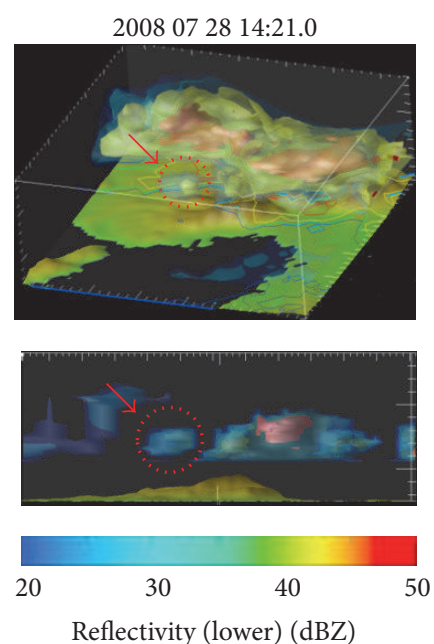
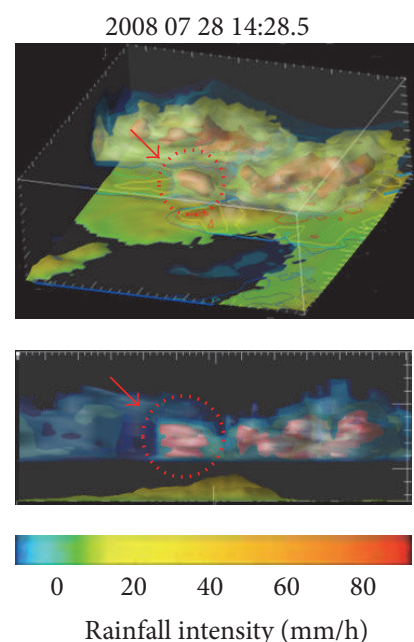
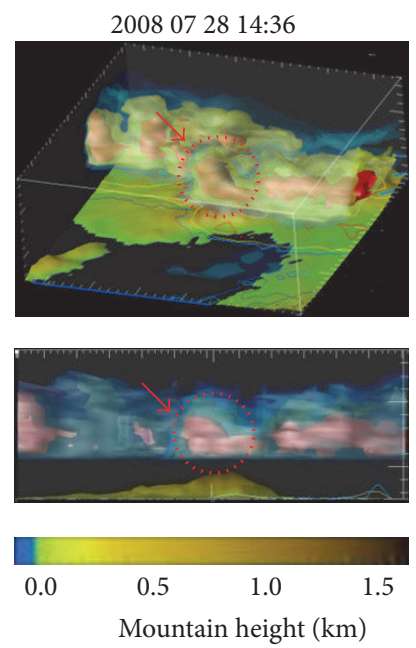

FIGURE 3: Three-dimensional reflectivity detected by the Miyama C-band radar operated by the MLIT. Contour lines of rainfall intensity are described on the ground. Toga River is located at the center of each figure.

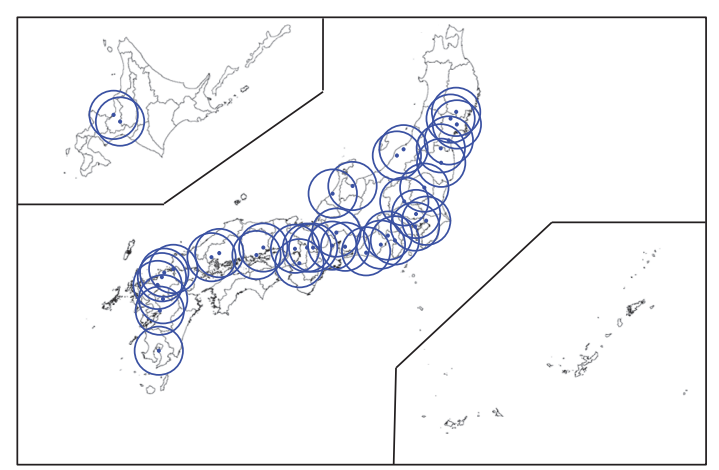

$80 \mathrm{~km}$ radius

FIGURE 4: Distribution of all radars in XRAIN and their detection areas. The maximum detecting radius of each radar is $80 \mathrm{~km}$.

observation data from the four radars in the Kinki area, the largest one is selected as the representative vorticity. With this methodology, approximate vorticity can be obtained. The vorticity criterion is set to be $0.03 \mathrm{~s}^{-1}$, which denotes that vorticity with a value greater than $0.03 \mathrm{~s}^{-1}$ is considered as our target in a rain-cell in the early stage. Special attention is given such that even if the real vorticity is unavailable, the approximate one is applicable to the purpose of qualitative identification of the danger level of a baby-rain-cell.

In the next section, we introduce the evaluation of the largest vertical vorticity for detection of a hazardous rain-cell.

\subsubsection{Evaluation of the Efficiency of Vorticity. A Guerrilla-} heavy rainfall is defined as a rainstorm generated by an isolated cumulonimbus cloud with an intensity of more than $50 \mathrm{~mm} / \mathrm{h}$ within $30 \mathrm{~min}$ after rainfall reaches the ground. To evaluate the efficiency of vorticity for identification of the danger level, we investigated 35 Guerrilla-heavy rainfall events, including the ones in Nakakita et al. [14], from 2010 to 2013. Details of the collected events are tabulated in Table 2, including occurrence date, time of first echo detection, first time of detection of vorticity greater than or equal to $0.03 \mathrm{~s}^{-1}$, and time and maximum rainfall intensity on the ground. All the events had vertical vorticity in the early stages before severe rainfall reached the ground.

Figure 8(a) shows statistics of the elapsed time since the first echo of a rain-cell was detected. Green bars denote the time interval between the vorticity detection and the first echo detection; the average time was $1.7 \mathrm{~min}$. Orange bars represent the time interval between the maximum rainfall intensity observation and the first radar echo detection; the average time was $25.3 \mathrm{~min}$. By average values, vorticity was detected 23.6 min earlier than the time at which maximum rainfall intensity occurred. As is shown in Figure 8(a), special attention was given such that, in our collected events, there was no event where vorticity and maximum rainfall intensity were detected simultaneously. In addition, Figure 8(b) illustrates the interval between the time of maximum rainfall intensity and vorticity detection. We found that the time at which the vorticity was detected was at least 10 min earlier than time at which the Guerrilla-heavy rainfall reached the ground. From this, vertical vorticity is proven to be an effective index for detection of a hazardous rain-cell and, further, for a Guerrilla-heavy rainfall prediction system, as is introduced in the next subsection.

\subsection{Development of Guerrilla-Heavy Rainfall Prediction Sys-} tem. Vorticity is an effective index to identify hazardous rain-cells that may develop into a severe storm leading to a Guerrilla-heavy rainfall. Based on this result, we developed a Guerrilla-heavy rainfall prediction system that include three major steps in the algorithm [14]: (1) detection of a babyrain-cell in the early stage of a rainstorm, (2) identification of the initial danger level of baby-rain-cells using estimated vorticity, and (3) three-dimensional tracking of the rain-cell until it develops into a rainstorm. The methodologies of early 


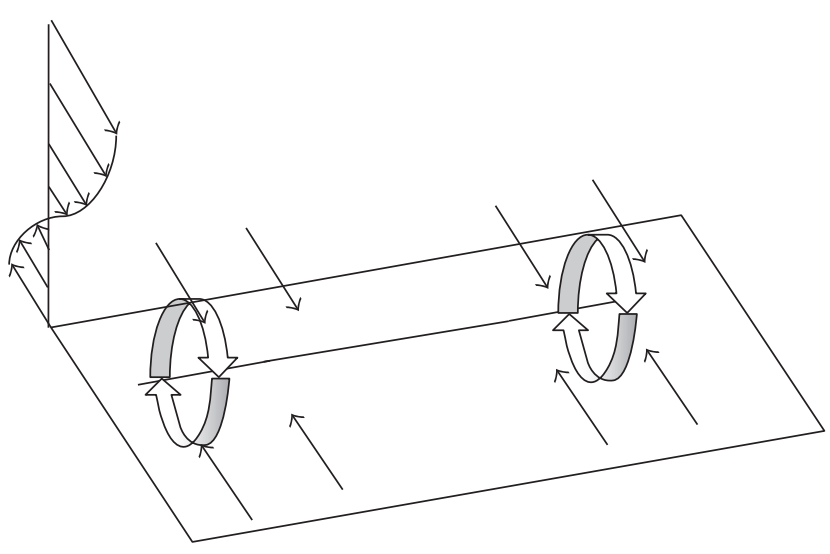

(a)

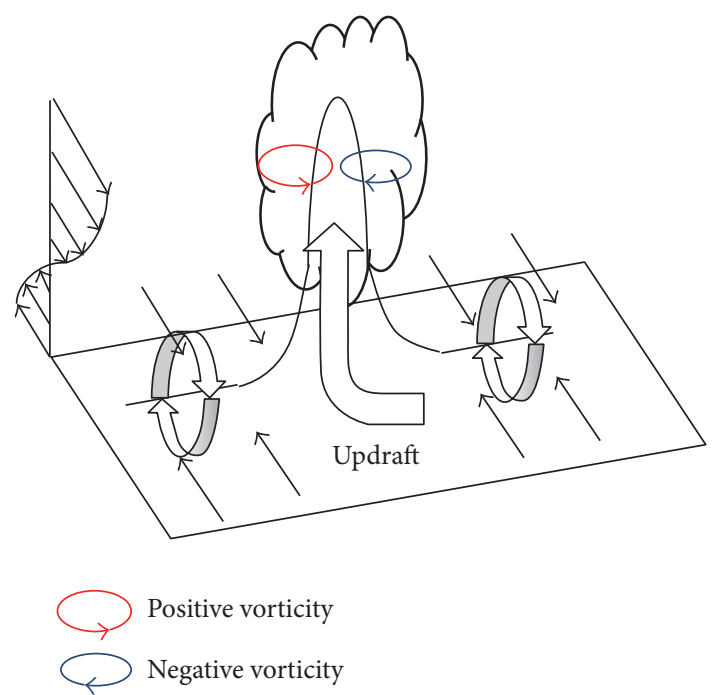

(b)

FIGURE 5: Schematic figures of formation of a vertical vortex tube. Circular arrows show circulations of horizontal vortex tube. (a) The vertical shear of horizontal wind generates a horizontally oriented vortex tube. (b) Updraft tilts a horizontal vortex tube into a vertical one (reproduced based on [15-17]).

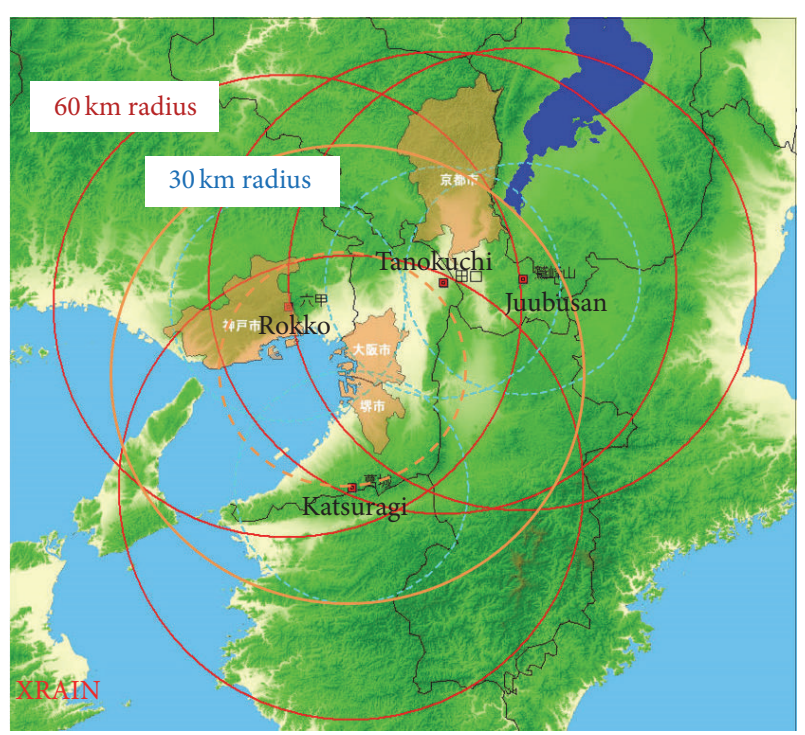

FIgURE 6: Four X-band radars in the Kinki area.

detection and identification of a baby-rain-cell have been described above.

The algorithm identifies rain-cells in three steps and classifies them under five danger levels (L1-L5) in different colors, as is shown in Figure 9. In the first step (1) a volume threshold, ranging from $0.125 \mathrm{~km}^{3}$ to $62.5 \mathrm{~km}^{3}$, is utilized to search baby-rain-cells. In the prediction system, rain-cells with volumes less than $0.125 \mathrm{~km}^{3}$ or greater than $62.5 \mathrm{~km}^{3}$ are not considered baby-rain-cells and are excluded.

The second step (2) is to identify initial baby-rain-cells by using a vorticity criterion of $0.03 \mathrm{~s}^{-1}$. Baby-rain-cells that do not include vorticity greater than or equal to the criterion are considered less dangerous and marked as L1 (blue); otherwise, baby-rain-cells with vorticity greater than or equal to the criterion are potentially dangerous and marked as L2 (yellow).

After identifying baby-rain-cells, the final step is to continually track the generated rain-cells and to identify their danger level of time variation. After being classified into the initial danger levels for baby-rain-cells (L1 and L2), the rain-cells are identified in the next time step using other danger levels, L3 (red), L4 (aqua), and L5 (gray). The vorticity criterion of $0.03 \mathrm{~s}^{-1}$ is still utilized to determine the danger level. Rain-cells with vorticities greater than or equal to this criterion are considered very dangerous and marked as L3. Rain-cells that do not include vorticity greater than or equal to the criterion are considered less dangerous and marked as L4. Finally, the remaining rain-cells are not the tracking targets and they are marked as L5.

To track hazardous cells, we propose an algorithm for rain-cell-tracking [14] which is introduced as follows. As the maximum moving speed of a rain-cell is around $60 \mathrm{~km} / \mathrm{h}$, the maximum moving range in $5 \mathrm{~min}$ is about $5 \mathrm{~km}$. Therefore, for all marked cells, a rain-cell-tracking algorithm, based on the connected component labeling (CCL) scheme [19], is performed to find the nearest cell within the moving range of $5 \mathrm{~km}$ in the next time step.

Figure 9 demonstrates one example of prediction. In the three-dimensional figures in the upper part, a new babyrain-cell is initially detected at 16:10 and marked as L1 (blue) because its vorticity was less than the vorticity criterion. In the next time step, at 16:15, the baby-rain-cell was identified as a dangerous cell and marked as L3 (red). As is shown in Figure 9, the baby-rain-cell was detected before the rainfall reached the ground at 16:10. Furthermore, at 16:15, the prediction system had already identified the baby-rain-cell 


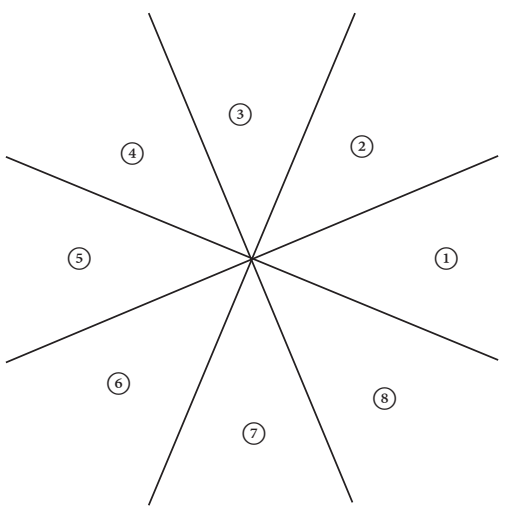

(a)

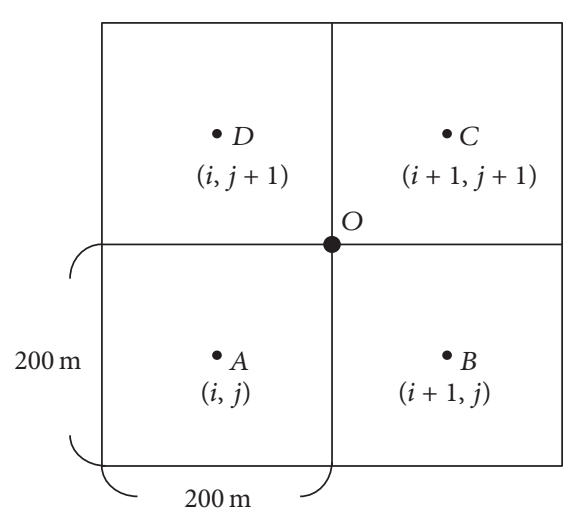

(b)

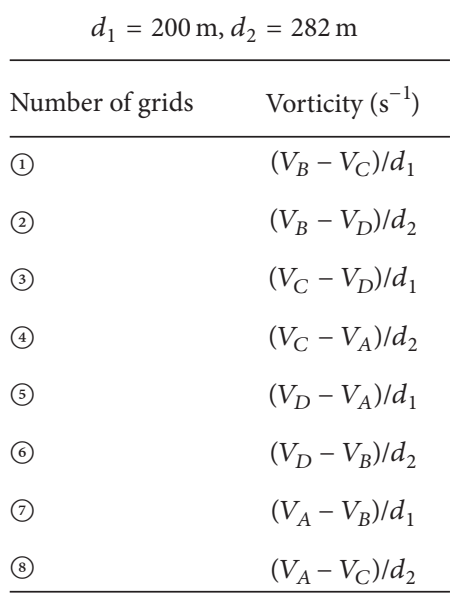

(c)

FIGURE 7: (a) Eight-direction map from the radar site, (b) grid number and corresponding formulas for vorticity calculations, and (c) vorticity estimation method for each direction.

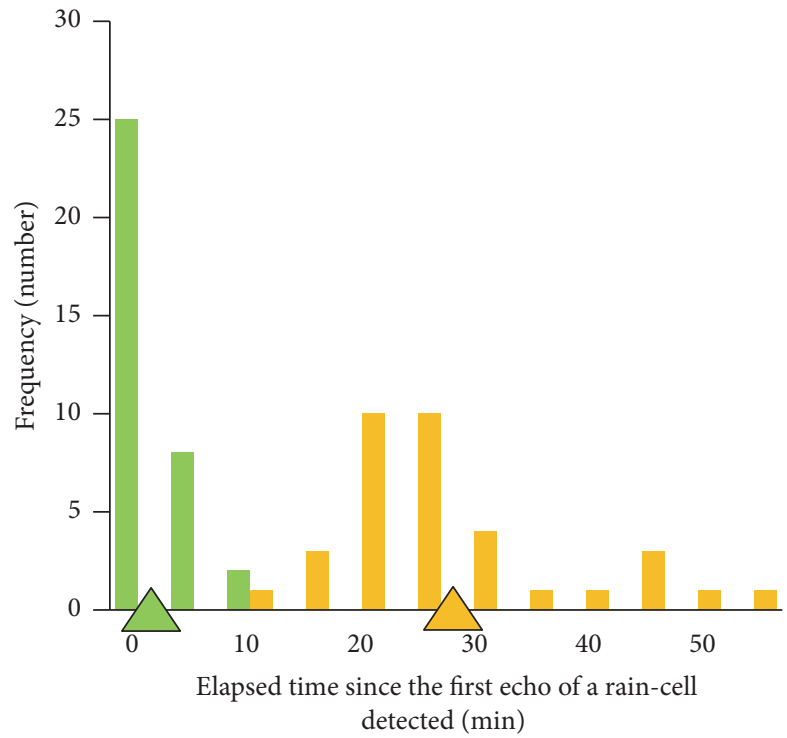

Vorticity detection (average $1.7 \mathrm{~min}$ )

Maximum intensity (average $25.3 \mathrm{~min}$ )

(a)

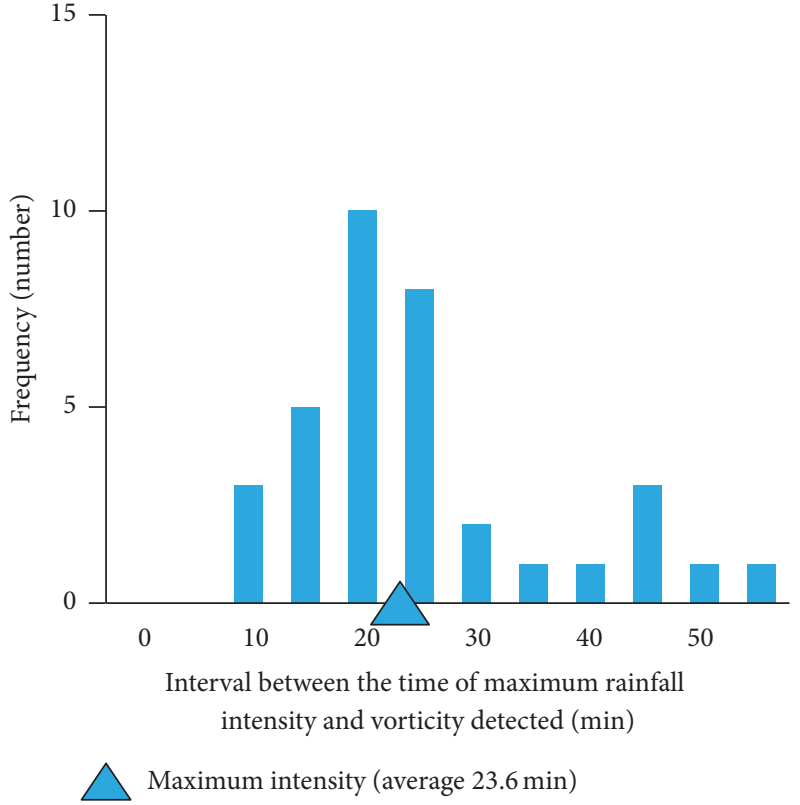

(b)

FiguRE 8: The statistics of the elapsed time since the first echo of a rain-cell was detected: (a) green bars: the time interval between the vorticity detection and the first radar echo detection; orange bars: the time interval between the maximum rainfall intensity observation and the first echo detection; (b) blue bars: the time interval between the maximum rainfall intensity observation and the vorticity detection.

as hazardous when slight rainfall appeared on the ground. This example proves that the prediction system can detect and identify a hazardous rain-cell in its early stage before a heavy rainfall reaches the ground.

To sum up, we showed that all rain-cells that caused Guerrilla-heavy rainfall had vertical vorticity values greater than or equal to $0.03 \mathrm{~s}^{-1}$ from the case study [14]. Based on this result, we developed a Guerrilla-heavy rainfall prediction system that does not miss predicting hazardous rain-cells [14]. As no hazardous rain-cell is missed in the prediction, the system is quite helpful for disaster prevention. Therefore, this pioneering and practical system has been adapted by the MLIT and implemented at five radar stations of XRAIN in the Kinki area [20]. One example in real practice is illustrated in Figure 10. However, not all baby-cells with large vertical vorticity values develop into severe storms leading to Guerrilla-heavy rainfalls, but in our system, these raincells will also be identified as hazardous rain-cells. In the recent research [21], the system has $17.1 \%$ false prediction rate over 70 events. For real practice, this rate of false prediction 
TABLE 2: List of Guerrilla-heavy rainfall events used for vorticity evaluation. This includes occurrence date, time of first echo detection, first time of detection of vorticity greater than or equal to $0.03 \mathrm{~s}^{-1}$, and time and maximum rainfall intensity on the ground.

\begin{tabular}{|c|c|c|c|c|c|}
\hline Date & $\begin{array}{c}\text { Time of rainfall on } \\
\text { the ground }\end{array}$ & $\begin{array}{c}\text { Time of } \\
\text { baby-cell-detection }\end{array}$ & $\begin{array}{c}\text { Time of vorticity } \\
\text { detection }\end{array}$ & $\begin{array}{c}\text { Time of maximum } \\
\text { intensity }\end{array}$ & $\begin{array}{l}\text { Maximum intensity } \\
(\mathrm{mm} / \mathrm{h})\end{array}$ \\
\hline $2010 / 7 / 7$ & $12: 37$ & $12: 35$ & $12: 35$ & 13:17 & 97.3 \\
\hline 2010/7/16 & $16: 27$ & $16: 15$ & $16: 15$ & $16: 55$ & 97.8 \\
\hline 2010/7/17 & $15: 58$ & $15: 50$ & $15: 50$ & $16: 33$ & 113.1 \\
\hline $2010 / 7 / 24$ & $16: 53$ & $16: 50$ & $16: 50$ & $17: 17$ & 116.9 \\
\hline $2010 / 8 / 6$ & $15: 44$ & $15: 40$ & $15: 40$ & $16: 05$ & 70 \\
\hline $2010 / 8 / 14$ & $12: 23$ & $12: 20$ & $12: 20$ & $12: 35$ & 107 \\
\hline $2010 / 8 / 20$ & $17: 55$ & $17: 55$ & $17: 55$ & $18: 13$ & 52.6 \\
\hline $2010 / 8 / 20$ & $18: 28$ & $18: 30$ & $18: 30$ & 19:15 & 66.3 \\
\hline $2010 / 8 / 24$ & $16: 31$ & $16: 30$ & $16: 30$ & $17: 21$ & 88.2 \\
\hline $2010 / 8 / 24$ & $16: 59$ & $16: 55$ & $16: 55$ & $17: 17$ & 82.7 \\
\hline $2010 / 8 / 26$ & $16: 52$ & $16: 50$ & $16: 55$ & $17: 17$ & 74.7 \\
\hline $2010 / 8 / 29$ & $16: 10$ & $16: 10$ & $16: 10$ & $16: 42$ & 79.3 \\
\hline $2010 / 8 / 30$ & $14: 57$ & $14: 55$ & $14: 55$ & $15: 43$ & 106.1 \\
\hline $2010 / 8 / 31$ & $15: 21$ & $15: 15$ & $15: 20$ & $15: 45$ & 111.8 \\
\hline $2011 / 7 / 24$ & $16: 24$ & $16: 25$ & $16: 25$ & $16: 39$ & 93.3 \\
\hline $2011 / 7 / 26$ & $14: 33$ & $14: 35$ & $14: 35$ & $14: 55$ & 60.8 \\
\hline $2011 / 7 / 28$ & $10: 18$ & $10: 20$ & $10: 25$ & $10: 41$ & 75.4 \\
\hline $2011 / 7 / 31$ & $16: 42$ & $16: 40$ & $16: 45$ & $17: 04$ & 78.7 \\
\hline $2012 / 7 / 20$ & $12: 36$ & $12: 40$ & $12: 40$ & $13: 03$ & 88.0 \\
\hline $2012 / 7 / 20$ & $13: 03$ & 13:05 & 13:05 & $13: 25$ & 79.2 \\
\hline $2012 / 7 / 22$ & $14: 26$ & $14: 25$ & $14: 25$ & $14: 45$ & 67.6 \\
\hline $2012 / 7 / 22$ & $16: 06$ & $16: 10$ & $16: 10$ & $16: 35$ & 114.4 \\
\hline $2012 / 7 / 25$ & $16: 08$ & $16: 15$ & $16: 15$ & $16: 35$ & 82.5 \\
\hline $2012 / 7 / 26$ & $15: 50$ & $15: 55$ & $15: 55$ & $16: 14$ & 78.0 \\
\hline $2012 / 8 / 11$ & $15: 58$ & $15: 55$ & $16: 05$ & $16: 17$ & 90.8 \\
\hline 2012/8/17 & $16: 44$ & $16: 40$ & $16: 40$ & $16: 59$ & 79.9 \\
\hline 2012/8/17 & $17: 23$ & $17: 25$ & $17: 30$ & $17: 44$ & 84.1 \\
\hline $2012 / 8 / 21$ & $16: 01$ & $16: 00$ & $16: 05$ & $16: 22$ & 88.7 \\
\hline $2012 / 8 / 23$ & $14: 54$ & $15: 05$ & $15: 05$ & $15: 31$ & 91.3 \\
\hline $2012 / 8 / 31$ & $15: 20$ & $15: 20$ & $15: 20$ & $15: 30$ & 103.2 \\
\hline $2012 / 8 / 31$ & $15: 32$ & $15: 30$ & $15: 40$ & $15: 47$ & 90.2 \\
\hline $2013 / 8 / 6$ & $13: 10$ & $13: 10$ & $13: 10$ & $13: 33$ & 78.9 \\
\hline $2013 / 8 / 6$ & $13: 45$ & $13: 45$ & $13: 50$ & $14: 05$ & 80.8 \\
\hline $2013 / 8 / 6$ & $16: 12$ & $16: 10$ & $16: 15$ & $16: 23$ & 54.8 \\
\hline $2013 / 8 / 7$ & $16: 45$ & $16: 45$ & $16: 45$ & $17: 10$ & 87.8 \\
\hline
\end{tabular}

is considered to be low and acceptable. Although this is an overestimation of hazardous rain-cells, from the viewpoint of disaster prevention, the fact that there are no missing predictions ensures the safety of people. To the best of our knowledge, this localized torrential rainfall prediction system is the safest in the world.

\section{An Analysis of Vortex Tube Structure}

As is mentioned in the Section 3, vertical vorticity values can be used to successfully identify hazardous baby-rain-cells aloft before they cause Guerrilla-heavy rainfalls. However, the reason why the vorticity is effective in identifying hazardous baby-rain-cells is still unclear. Hence, we carried out an analysis of vorticity inside rain-cells that caused Guerrillaheavy rainfall in order to resolve the relationship between baby-rain-cell growth and corresponding vorticity behavior. Additionally, improved understanding of baby-rain-cell development facilitates quantification of heavy rainfall risk.

As indicated in Section 3.1, vortex tube tilting is the characteristic phenomenon of early stage supercell development. In an analysis of rain-cells in 35 events that did not develop into a supercell, Nakakita et al. [14] found vorticity values greater than or equal to $0.03 \mathrm{~s}^{-1}$. Based on this result, we 

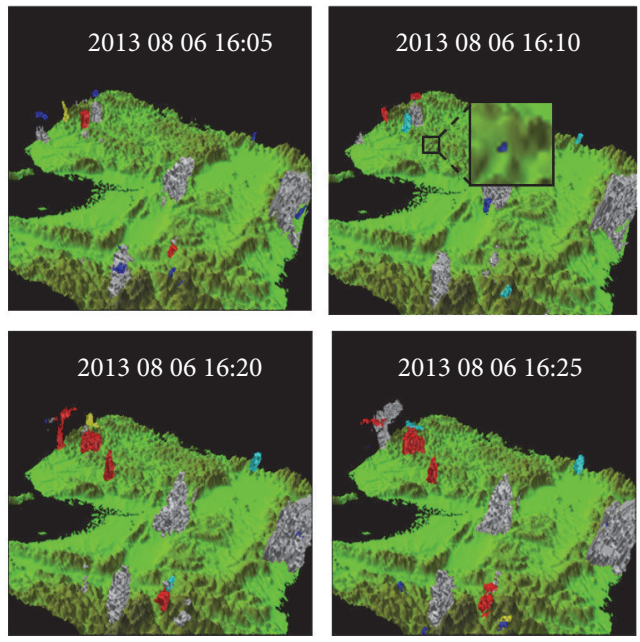

(a)
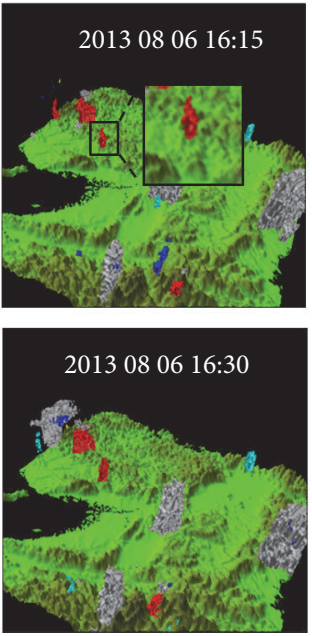

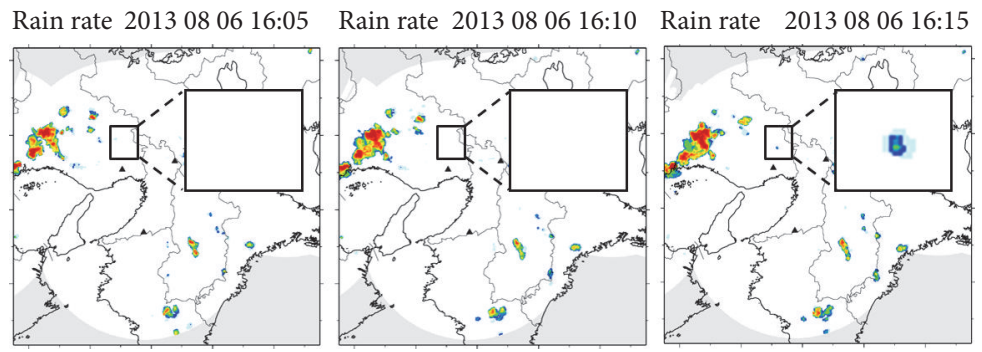

Rain rate 20130806 16:20 Rain rate 20130806 16:25 Rain rate 20130806 16:30

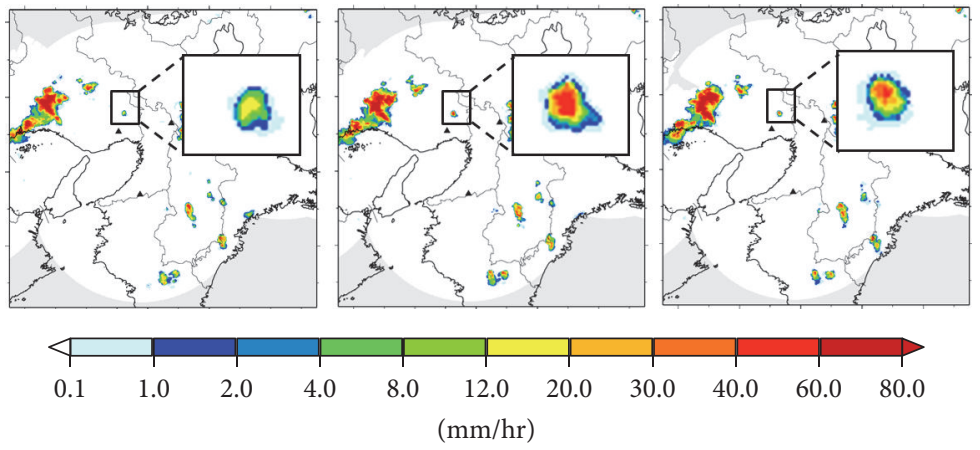

(b)
L1 less dangerous baby-rain-cell L2 likely dangerous baby-rain-cell L3 dangerous baby-rain-cell L4 likely safe baby-rain-cell L5 others

(c)

FIGURE 9: Risk prediction of a baby-rain-cell using the Guerrilla-heavy rainfall system: three-dimensional figures (a), two-dimensional rainfall intensity figures (b), and legends of each danger level (c).

assume that the growth of a baby-rain-cell is similar to that of a supercell. To verify this assumption, we analyzed the vorticity distribution inside baby-rain-cells for 16 selected Guerrilla-heavy rainfall events. In the following, we describe the data processing methodology, vorticity distribution analysis, and verification of vortex tube behavior inside babyrain-cells.

4.1. Methodology of Data Processing. As our focus is on analysis of the vorticity structure in baby-rain-cells, obtaining an accurate vorticity distribution is crucial. In our study, we used observed reflectivity and Doppler velocity. To focus on accurate vorticity distribution, we propose a new data processing methodology. In what follows, we shall present the new methodology and compare it with conventional methodology [14].

(1) Coordinate System Transformation. In the analysis by Nakakita et al. [18], as the original data is in the polar coordinate system, an interpolation method was utilized to transform the polar coordinate system into the threedimensional Cartesian coordinate system. For reflectivity and Doppler velocity, the $(x, y, z)$ dimensions of a unit element are $500 \mathrm{~m} \times 500 \mathrm{~m} \times 250 \mathrm{~m}$ and $200 \mathrm{~m} \times 200 \mathrm{~m} \times 200 \mathrm{~m}$, respectively. Meantime, as the duration of one cycle of radar volume scan is five minutes, the analysis time interval was also set to be five minutes. However, we considered this methodology to be applicable to the present study, because 

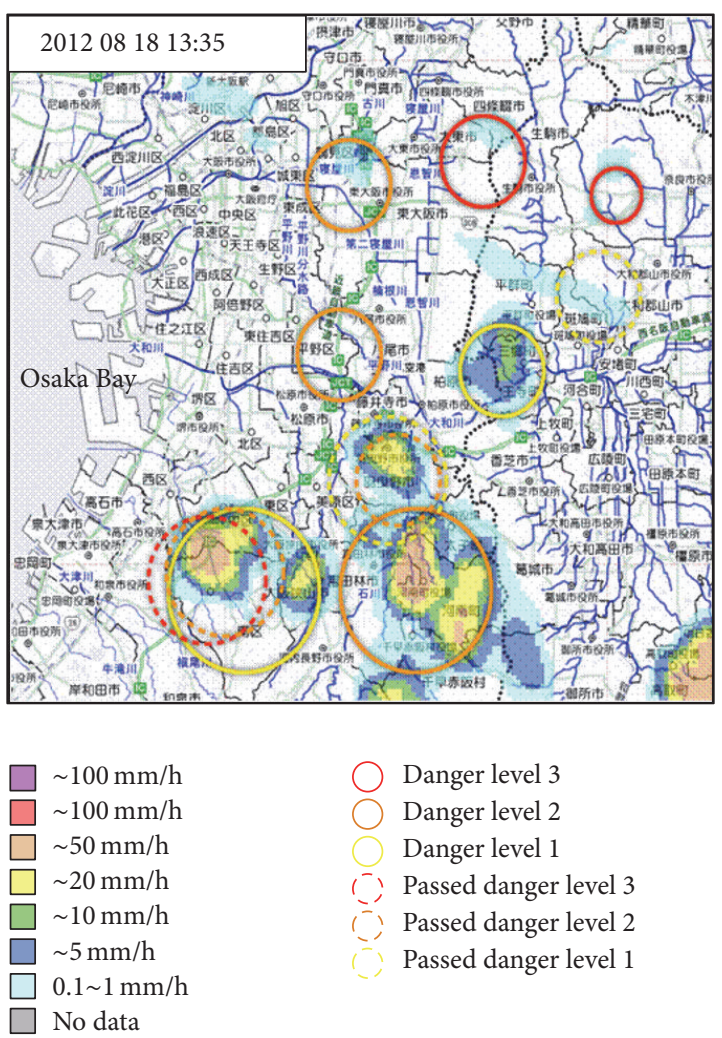

FIGURE 10: One example of the results using the Guerrilla-heavy rainfall prediction system by radars of the MLIT [20]. Circles in different colors denote the dangerous range and the corresponding danger level.

the spatial resolution of data is too low to analyze small and rapidly growing baby-rain-cells, and interpolation may cause artificial distortion of data. Therefore, to obtain precise vorticity distribution, we utilized a new methodology of data processing for extraction of the vorticity distribution from Doppler velocity.

Raw data in the polar coordinate system was directly used for vorticity frequency analysis, without interpolation. Note that, for figure illustrations and vortex tube analysis, we used the plane projection method to convert PPI scan data to planar data in the two-dimensional Cartesian coordinate system. Also for detailed display, the spatial resolution in each plane is $50 \mathrm{~m}$ by $50 \mathrm{~m}$. With data in the high spatial resolutions, we can accurately analyze the behavior of a small rain-cell.

(2) Noise Data Threshold. The threshold of noise data is critical in radar data processing to avoid interruption from noise echoes. In conventional study, the threshold is set at $20 \mathrm{dBZ}$ for reflectivity data. However, as we focused on the very early stage of small rain-cell development, the reflectivity threshold was set at $5 \mathrm{dBZ}$, and Doppler velocity was directly used without being filtered by the reflectivity threshold. To distinguish real echoes from noise, we manually collected the first echo data by visual judgment of the reflectivity distribution and Doppler velocity.

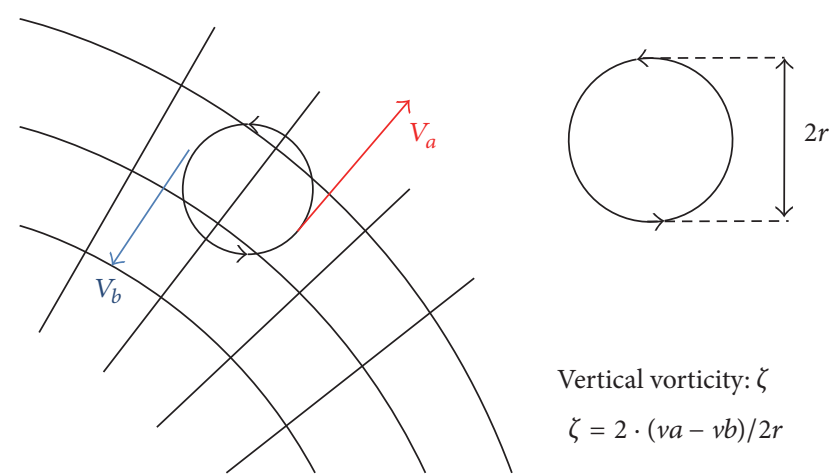

FIGURE 11: Formulas for vorticity calculation, which utilizes adjacent meshes of Doppler velocity data (Nakakita et al., [22]).

(3) Vorticity Calculation Method. We introduced an eightdirection classification and used eight different equations for calculating approximate vorticity [18], as shown in Figure 7. If varying vorticity values are obtained from different radar directions, we suggest selecting the largest positive vorticity value at a location to identify hazardous rain-cells of which the vorticity value is greater than or equal to $0.03 \mathrm{~s}^{-1}$. We introduced a new methodology in the present study to obtain a more accurate vorticity distribution. As is shown in Figure 11, vorticity is calculated by the adjacent meshes in Doppler velocity data in the polar coordinate system. It should be noted that our focus was to analyze vortex behavior in three-dimensions, so both positive and negative vorticity were important for analysis. So, in the vorticity distribution we kept all positive and negative values of vorticity at each location without selecting the greatest positive value. Furthermore, the criterion of absolute value of vorticity is set as $0.01 \mathrm{~s}^{-1}$. With this value, which is much smaller than the conventional criterion $0.03 \mathrm{~s}^{-1}$, we were able to analyze the relatively low vorticity of a very early stage rain-cell.

Figure 12 illustrates examples of the data processing output for reflectivity, Doppler velocity, and vorticity distribution. Contour lines show height above the ground, and the interval between major contour lines is $1,000 \mathrm{~m}$.

4.2. Vorticity Distribution Analysis. We collected 16 Guerrilla-heavy rainfall events in 2013 and 2014 to analyze the vorticity distribution. Details of the events are tabulated in Table 3, including occurrence date, time of first echo detection, first time of rainfall recorded on the ground, and time of maximum rainfall intensity. For each event, the count of vorticity is calculated using the processed data in the polar coordinate system. By summarizing the count and absolute value of vorticity in all baby-rain-cells, Figure 13 shows the frequency distribution of absolute value of vorticity in the first echo of all collected events. In the baby-rain-cells that did not form supercells, clearly, both positive and negative vorticity existed. And according to this figure, the positive vorticity frequency was greater than negative (Figure 13). This may be due to large-scale positive vorticity in the background affecting small-scale vorticity in the first echoes. However, we focused on only qualitative analysis of the existence 
TABLE 3: List of the Guerrilla-heavy rainfall events used for analysis of vorticity and vortex tubes. This includes occurrence date, time of first echo detection, first time of rainfall recorded on the ground, and time of maximum rainfall intensity.

\begin{tabular}{lcccc}
\hline Event & Date & Time of baby-cell-detection & Time of rainfall on the ground & Time of maximum intensity \\
\hline 1 & $2013 / 8 / 6$ & $13: 03$ & $13: 10$ & $13: 33$ \\
2 & $2013 / 8 / 6$ & $13: 42$ & $13: 45$ & $14: 05$ \\
3 & $2013 / 8 / 6$ & $16: 06$ & $16: 10$ & $16: 23$ \\
4 & $2013 / 8 / 7$ & $16: 40$ & $16: 45$ & $17: 10$ \\
5 & $2014 / 8 / 17$ & $9: 12$ & $9: 13$ & $9: 39$ \\
6 & $2014 / 8 / 17$ & $12: 08$ & $12: 09$ & $12: 23$ \\
7 & $2014 / 8 / 17$ & $15: 47$ & $15: 50$ & $16: 04$ \\
8 & $2014 / 8 / 18$ & $15: 58$ & $16: 04$ & $16: 18$ \\
9 & $2014 / 8 / 23$ & $11: 46$ & $11: 52$ & $12: 08$ \\
10 & $2014 / 8 / 23$ & $11: 38$ & $11: 53$ & $12: 05$ \\
11 & $2014 / 8 / 23$ & $15: 57$ & $16: 06$ & $16: 29$ \\
12 & $2014 / 8 / 23$ & $16: 28$ & $16: 34$ & $16: 57$ \\
13 & $2014 / 8 / 24$ & $12: 33$ & $12: 38$ & $12: 53$ \\
14 & $2014 / 8 / 25$ & $12: 20$ & $12: 26$ & $12: 49$ \\
15 & $2014 / 8 / 25$ & $17: 33$ & $17: 37$ & $17: 51$ \\
16 & $2014 / 8 / 27$ & $16: 25$ & $16: 38$ & $17: 13$ \\
\hline
\end{tabular}

or absence of negative vorticity in baby-rain-cells. We will analyze the data on different frequencies of positive and negative in further work.

Vertical vortex tubes were identified by connecting vorticity distributions at different altitudes, and we found they were present in all baby-rain-cells that did not evolve into supercells. Figure 14 demonstrates one example of vertical vortex tube structure from a rain-cell in one of the collected events. However, more evidence is needed to support our assumption. Hence, we estimated updraft region and analyzed the relationship between vorticity and updraft by a real event. We shall present the methodology and results in the next section.

\subsection{Analysis of the Relationship between Vortex Tubes and Updraft}

4.3.1. Theoretical Vortex Tube Behavior in the Early Stage of a Supercell. In recent decades, vortex tube structure has been investigated to clarify the supercell development process, for example, [15-17], but, to our knowledge, systematic analysis of rain-cell vorticity distribution has not been investigated to date, except for supercells. This research is a pioneering study to analyze vortex tube structure at high spatial resolution, associated with a severe Guerrilla-heavy rainfall storm.

Consider a horizontally oriented vortex tube induced by the vertical shear of a horizontal environmental wind which flows perpendicular to the vortex tube, as is shown in Figure 5. Vertically oriented vortex tubes motion can be expressed by the vorticity equation in the $z$-direction [17] as

$$
\frac{d \zeta}{d t}=\xi \cdot \frac{\partial w}{\partial x}+\eta \cdot \frac{\partial w}{\partial y}+\zeta \cdot \frac{\partial w}{\partial z}
$$

where $(\xi, \eta, \zeta)$ are vorticities in the $(x, y, z)$ directions, respectively, and $w$ is the $z$-component of velocity. In the right hand side (RHS) of (1), the first two terms represent the tilting motion of a horizontal vortex tube by a vertical shear of horizontal wind, and the third term represents the stretching motion of the vertical vortex tube due to variable $w$ in the $z$-axis direction. Vorticity motion is three-dimensional, and all components are nonlinearly coupled with each other, but for the sake of clear explanation only the vertical vorticity equation is presented here.

One example is given for explanation as follows. In an environment with vertical vorticity $\zeta=0$ when a vertical shear of horizontal wind occurs $\partial u / \partial z \neq 0$, the horizontal vortex tube of $\eta$ begins to be tilted by the updraft. Hence, according to (1), vertical vorticity $\zeta$ starts to be altered by the tilting motion through the second term in the RHS. In this condition, the $z$-direction vorticity equation can be rearranged into

$$
\frac{d \zeta}{d t}=\eta \cdot \frac{\partial w}{\partial y}=\frac{d \bar{u}(z)}{d z} \cdot \frac{\partial w}{\partial y}
$$

where $\bar{u}(z)$ is the velocity of an environmental wind. As is shown in Figure 5(b), from (2) a positive vorticity is generated in the left side of the updraft relative to the shear flow $(\partial w / \partial y>0)$, and simultaneously a negative vorticity appears in the right side of the updraft relative to the shear flow $(\partial w / \partial y<0)$ instead. Once the pair of vertical vorticities is generated, an updraft feedback mechanism amplifies vertically oriented vortex tube by a stretching motion, as represented by the third term in (1). From this theoretical explanation, we can conclude that a pair of positive and negative vortex tubes exists along the updraft. Further analysis is needed to verify this conclusions as discussed below. 


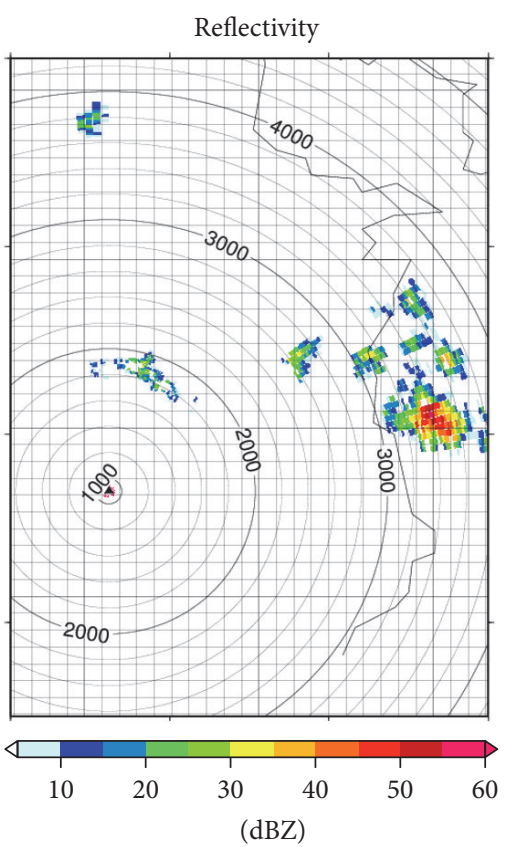

(a)

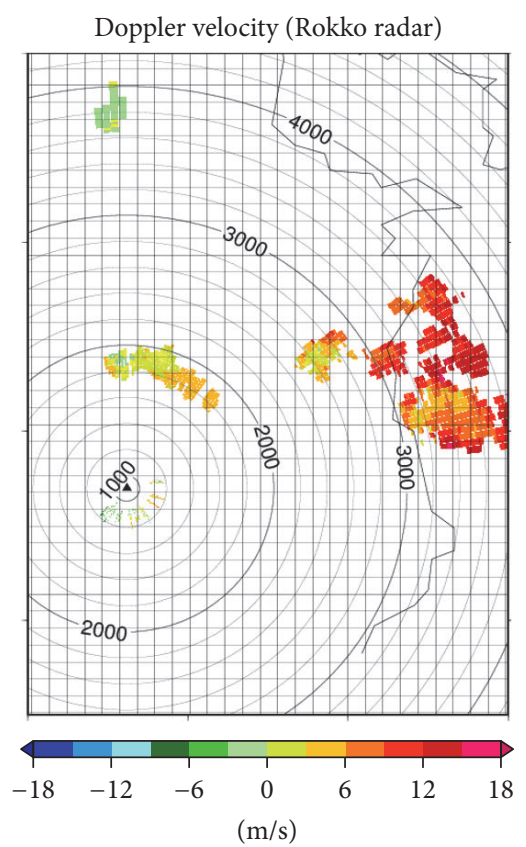

(b)

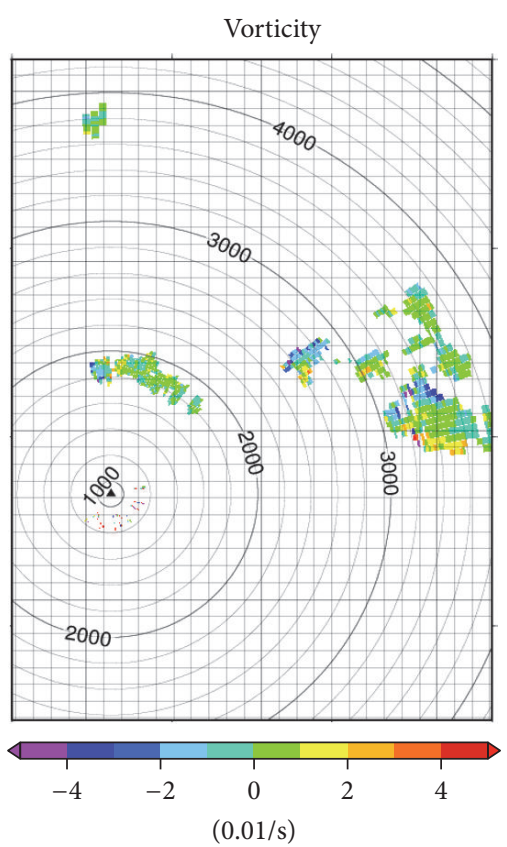

(c)

FIGURE 12: One example of distributions of reflectivity (a), Doppler velocity (b), and vorticity (c) after data processing.

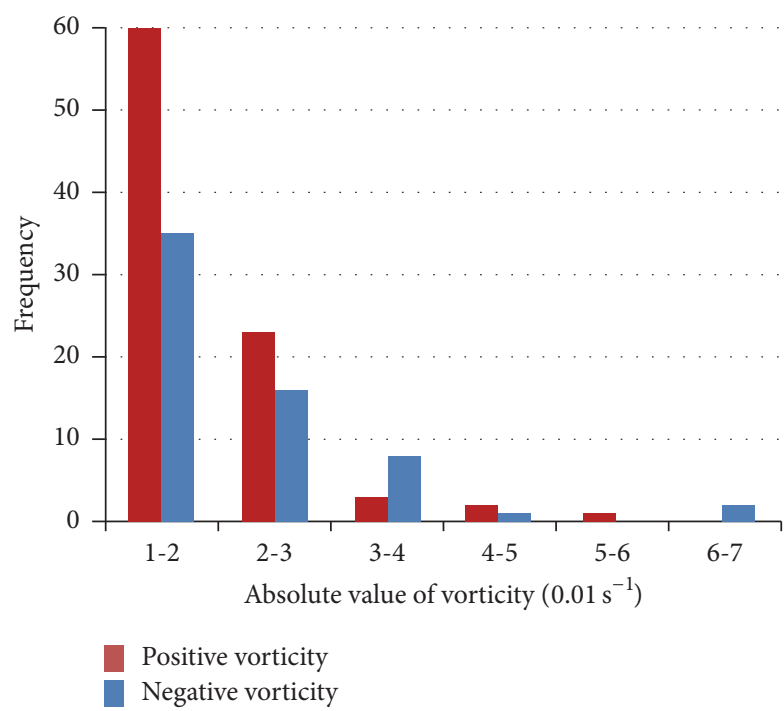

FIGURE 13: The frequency distribution of the absolute values of negative and positive vorticity in baby-rain-cells.

4.3.2. Methodology of Updraft Identification. Hereafter, the relationship between an updraft and corresponding vorticity location is examined in a real event. The importance of an updraft for developing a pair of positive and negative vortex tubes in the vertical direction has been explained in Section 4.3.1. In order to identify updraft location, we utilized differential reflectivity $\left(Z_{\mathrm{DR}}\right)$ and dual Doppler radar measurement.

(1) High $Z_{D R}$ Column. $Z_{\mathrm{DR}}$ is one of the polarimetric parameters, and many researchers have focused on $Z_{\mathrm{DR}}$ to identify

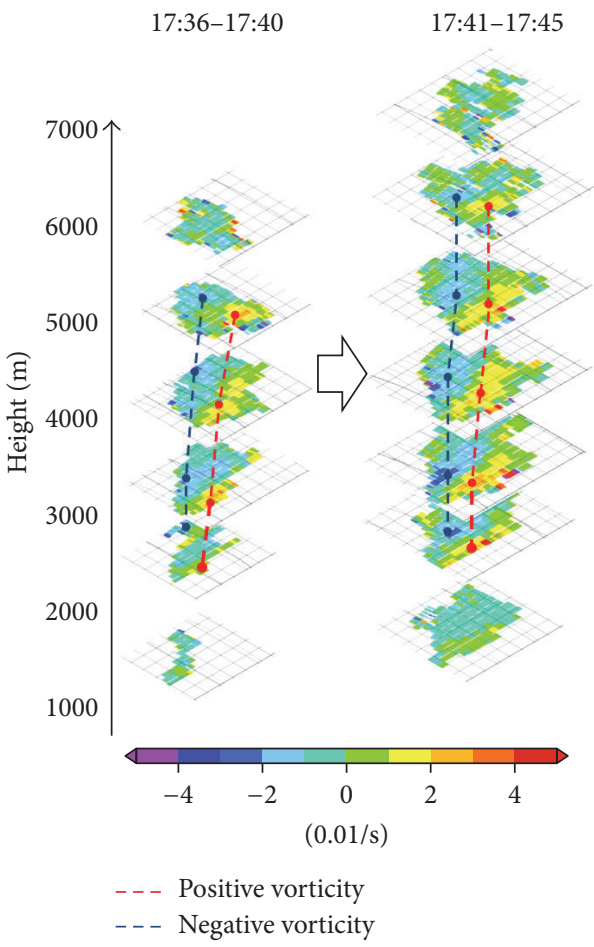

Figure 14: One example of structure of vertical vortex tube in the rain-cell of the event 15 in Table 3 . The red lines show positive vorticity and the blue lines show negative vorticity.

updraft location in thunderstorms, for example, [23-25]. $Z_{\mathrm{DR}}$ depends on the shape and size of hydrometeors; negative values of $Z_{\mathrm{DR}}$ can denote vertically oriented scatterers, while positive values denote horizontally oriented scatters, that is, 
raindrops. Generally, few raindrops exist above the freezing level, but with updraft some raindrops are lifted up above the freezing level; hence, a high $Z_{\mathrm{DR}}$ column can be observed above the freezing level. Thus, this high $Z_{\mathrm{DR}}$ column is an effective index to identify an updraft. As is mentioned in Section 2.2, $Z_{\mathrm{DR}}$ is corrected to minimize the attenuation problem using $K_{\mathrm{DP}}$ by the MLIT [11]. In addition to this, in our study, we carried out additional $Z_{\mathrm{DR}}$ correction using reflectivity. The concept of the correction and the calculation method are explained below. Small reflectivity near the ground indicates light rain, and then the raindrop shape must be round. As is mentioned above, $Z_{\mathrm{DR}}$ depends on the shape of hydrometeors, so it should show almost $0 \mathrm{~dB}$ when the target shape is round. However, actual observed $Z_{\mathrm{DR}}$ sometimes show larger (smaller) value than $0 \mathrm{~dB}$ when reflectivity is small value (greater than or equal to $5 \mathrm{dBZ}$ and less than $20 \mathrm{dBZ}$ ). Thus, we calculated the bias value by a leastsquare technique so as to minimize the sum of squared errors of $Z_{\mathrm{DR}}$. For further details refer to Nakakita et al. [22].

(2) Multiple Doppler Radar Analysis. The radars cannot observe vertical velocity; thus we estimated it with multiple Doppler radar analysis using the variational technique proposed by Shimizu and Maesaka [26]. This variational method minimizes a cost function $J$, defined as the sum of squared errors, in the entire analysis domain. In this study, the LBFGS method proposed by Liu and Nocedal [27] was used to optimize the cost function. The cost function is defined as follows:

$$
\begin{aligned}
J= & J_{o}+J_{d}, \\
J_{o}= & \frac{1}{2} \sum_{i, j, k, m} \lambda_{o} \\
& \cdot\left(V r_{m}-u \cos A-v \cos B-(w+w t) \cos C\right)^{2}, \\
J_{d}= & \frac{1}{2} \sum_{i, j, k} \lambda_{d} D^{2}, \\
A= & \frac{x_{m}}{r_{m}}, \\
B= & \frac{y_{m}}{r_{m}}, \\
C= & \frac{z_{m}}{r_{m}}, \\
D= & \frac{\partial \bar{\rho} u}{\partial x}+\frac{\partial \bar{\rho} v}{\partial y}+\frac{\partial \bar{\rho} w}{\partial z},
\end{aligned}
$$

where $J_{o}$ is the difference between observed and analyzed radial velocity, $J_{d}$ is continuity equation error, $m$ is the number of radars, $u, v$, and $w$ are velocity components of wind, $x_{m}, y_{m}$, and $z_{m}$ are the distance between the target and the radar site, $w_{t}$ is the target drop velocity, $\bar{\rho}$ is the mean horizontal air density, and $\lambda_{o}$ and $\lambda_{d}$ are reciprocals of the error variance. Here, the Cartesian coordinate system is used for reflectivity and Doppler velocity, where the dimension of a unit rectangular element is $1 \mathrm{~km} \times 1 \mathrm{~km} \times 500 \mathrm{~m}$. With these data, we estimated vertical velocity and identified the updraft region.

4.3.3. Analysis of Vortex Tube Tilting in the Rain-Cell. Event 15 of Table 3 was selected for updraft identification based on clear vortex tube shapes and high $Z_{\mathrm{DR}}$ above the freezing level. Figure 15 shows the (a) Doppler velocity observed by Tanokuchi radar and schematic diagram of tilting vortex tube, (b) vorticity calculated using Doppler velocity, (c) $Z_{\mathrm{DR}}$, and (d) vertical velocity computed by dual Doppler analysis using Katsuragi and Tanokuchi radar data. In this event, as the stronger west wind occurs at the high altitude, we can reasonably assume a horizontal vortex tube was generated due to vertical shear, as is shown in Figure 15(a).

From the theoretical analysis in Section 4.3.2, the west wind should produce positive vorticity in the south and negative in the north from the theoretical analysis in the previous paragraphs as is shown in Figure 15(a). As is shown in Figure 15(b), the property above is successfully testified by the vorticity distribution using Doppler velocity. And it is noticed that a pair of vorticities was existed in the babyrain-cell. We consider that an updraft can produce the pair of vortex tubes in the early stage of the rain-cell. The result reinforces the hypothesis that vortex tube development in rain-cells forming a Guerrilla-heavy rainfall is similar to that in the early stage of supercell.

4.3.4. Analysis of Updraft Location. Furthermore, since vortex tube structures have been shown to exist in rain-cells, an updraft must be present between the pair of positive and negative vorticity, as is shown in Figure 15(a). We verify updraft location based on a high $Z_{\mathrm{DR}}$ column location and multiple Doppler radar analysis. In this event, the bias value of $Z_{\mathrm{DR}}$ was $0.68 \mathrm{~dB}$. Thus, the bias value was subtracted from observed $Z_{\mathrm{DR}}$ and we used corrected value for the analysis.

A high $Z_{\mathrm{DR}}$ column can be extended above the freezing level by an updraft, for example, $[23-25]$. $Z_{\mathrm{DR}}$ greater than or equal to $2 \mathrm{~dB}$ above the freezing level, which was $5,100 \mathrm{~m}$ in this event, is used for identification of the updraft location. Zero degree height was estimated using the sonde observation data operated by the Japan Meteorological Agency (JMA) in Shionomisaki in the Kinki area. As a result, a high $Z_{\mathrm{DR}}$ column is found between positive and negative vorticities, as is shown in Figure 15(c). For multiple Doppler radar analysis, we used two radar observations' data from Tanokuchi and Katsuragi. As is shown in Figure 15(d), a strong updraft region is found between positive and negative vorticities. These results indicated that a pair of positive and negative vorticities coexists with an updraft in the rain-cell.

To sum up, through verification of vorticity distribution and updraft location with a real event, we have shown that vortex tube tilting, similar to that in a supercell, also exists in a rain-cell which forms a Guerrilla-heavy rainfall.

\section{Conclusion}

From the serious flood event in Toga River, we have realized that an additional period of more than five to ten minutes 


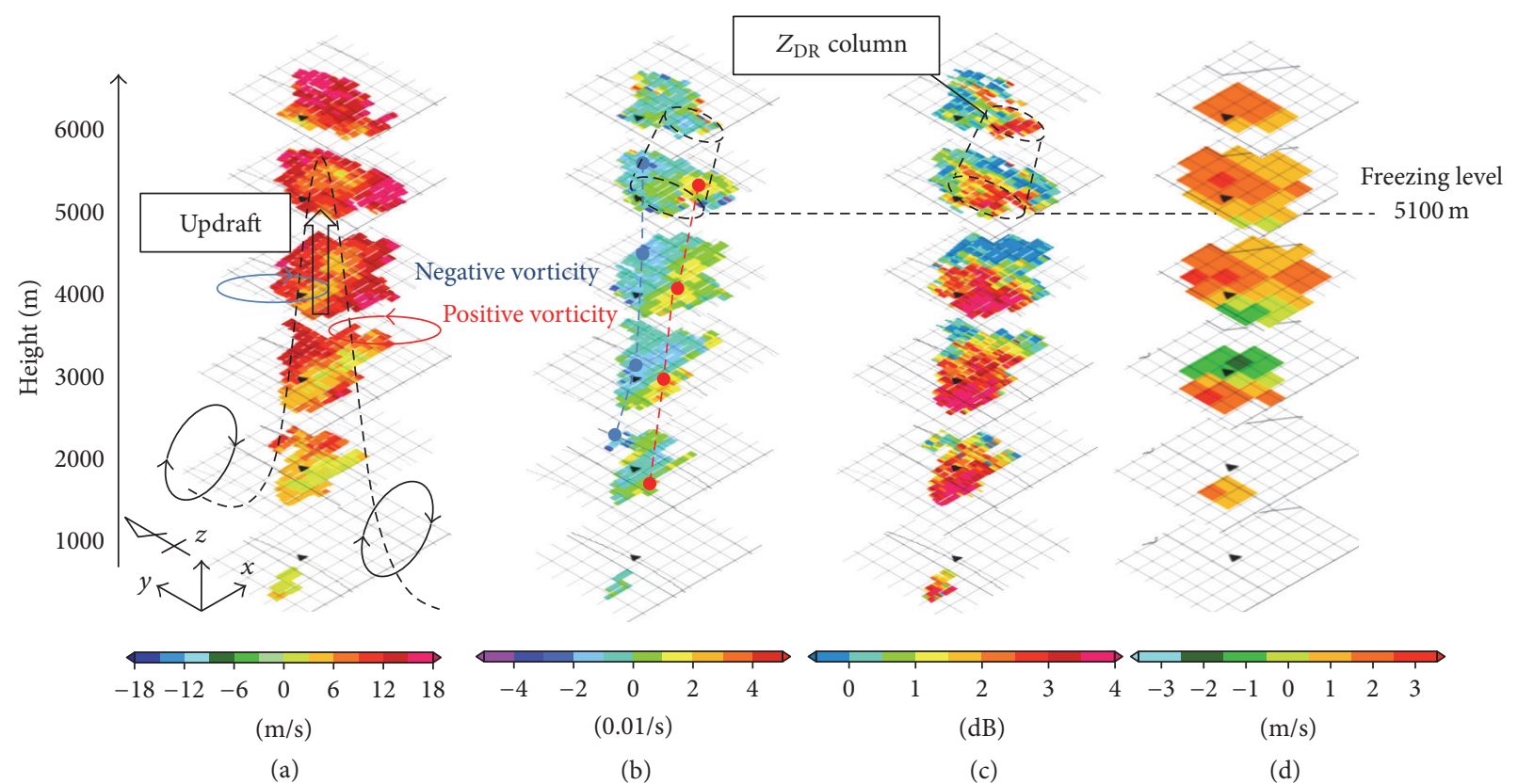

FIGURE 15: The feature of the event 15 observed by Tanokuchi radar; (a) Doppler velocity and schematic diagram of tilting vortex tube, (b) vorticity calculated using Doppler velocity data, (c) $Z_{\mathrm{DR}}$, and (d) vertical velocity computed with dual Doppler analysis using Katsuragi and Tanokuchi radar data. The columns described in black color show the region of the high $Z_{\mathrm{DR}}$ column $(\mathrm{b}, \mathrm{c})$.

is quite vital and helpful to save precious human lives by evacuating people from a dangerous river before a flood arrival. To obtain this important period, we have developed a system for detection of hazardous rain-cells at an earlier stage.

To protect human life, The most crucial objective for any disaster prevention system is not to miss a disaster prediction. In our analysis, we found that all rain-cells which brought hazardous Guerrilla-heavy rainfalls exhibited vertical vorticity values greater than or equal to $0.03 \mathrm{~s}^{-1}$. Therefore, to detect a Guerrilla-heavy rainfall, we utilized this vorticity criterion to develop a prediction system which would not miss prediction of any hazardous rain-cell. Regarding efficiency of real practical rainfall detection, this system has been implemented in five operating radar stations of XRAIN in the Kinki area.

Additionally, as a pioneering attempt, we also performed a brand new vorticity analysis of baby-rain-cells in the events of Guerrilla-heavy rainfalls. As a result, we verified the existence of vertical vortex tubes inside rain-cells which did not develop into supercells. Furthermore, vortex tube tilting motion, similar to that in a supercell, was also proven to exist in the rain-cell which formed a Guerrilla-heavy rainfall. These two results not only make a breakthrough of babyrain-cell analysis, but also help to clarify the importance of vorticity analysis for efficient identification of hazardous baby-rain-cells.

To better understand the developing mechanism of a baby-rain-cell, it is critical and necessary to analyze flow structure in a cumulonimbus cloud aloft in the atmosphere before raindrop generation. So, analysis of observation data from a cloud radar and a Light Detection and Ranging (Lidar) will be our future task. With better understanding, it is certain that the precise quantitative risk prediction system of Guerrilla-heavy rainfall could be realized, and perfect protection of human lives from flood disasters can be surely guaranteed in the near future.

\section{Competing Interests}

The authors declare that they have no competing interests.

\section{Acknowledgments}

The authors greatly acknowledge the financial support of JSPS KAKENHI (Grant nos. 22226010 and 15H05765). The valuable assistance and data from the Kasen-Sabo Research project by the Ministry of Land, Infrastructure, Transport and Tourism (MLIT) of Japan is also deeply appreciated.

\section{References}

[1] I. Fujita, "Disaster investigation of Toga River flood," in Proceedings of the Japanese Symposium of River Disaster, 2009 (Japanese).

[2] E. Nakakita, H. Yamabe, and K. Yamaguchi, "Tragic disaster due to very localized torrential rainfall occurred in Kobe Urban Area in Japan and installation of new radar network system for early detection of the storm system," U-City \& Hydroinformatics, World City Forum 2009, Sangdo Convensia, Incheon, Korea 18-21 August, 2009.

[3] E. Nakakita, H. Yamabe, and K. Yamaguchi, "Earlier detection of the origin of very localized torrential rainfall," Journal of Hydraulic Engineering, Japan Society of Civil Engineers, vol. 54, pp. 343-348, 2010 (Japanese). 
[4] C. A. Knight and L. J. Miller, "First radar echoes from cumulus clouds," Bulletin-American Meteorological Society, vol. 74, no. 2, pp. 179-188, 1993.

[5] C. A. Knight, J. Vivekanandan, and S. G. Lasher-Trapp, "First radar echoes and the early ZDR history of Florida cumulus," Journal of the Atmospheric Sciences, vol. 59, no. 9, pp. 1454-1472, 2002.

[6] F. Burnet and J.-L. Brenguier, "The onset of precipitation in warm cumulus clouds: an observational case-study," Quarterly Journal of the Royal Meteorological Society, vol. 136, no. 647, pp. 374-381, 2010.

[7] S. Göke, H. T. Ochs III, and R. M. Rauber, "Radar analysis of precipitation initiation in maritime versus continental clouds near the Florida coast: inferences concerning the role of $\mathrm{CCN}$ and giant nuclei," Journal of the Atmospheric Sciences, vol. 64, no. 10, pp. 3695-3707, 2007.

[8] V. Chandrasekar and A. P. Jayasumana, "Radar design and management in a networked environment," in Proceedings of the ITCom 2001: International Symposium on the Convergence of IT and Communications, pp. 142-147, International Society for Optics and Photonics, 2001.

[9] M. Maki, "X-band polarimetric radar network in the Tokyo metropolitan area-X-NET," in Proceedings of the 5th European Conference on Radar Meteorology, 2008.

[10] D. J. McLaughlin and V. Chandrasekar, "Short wavelength technology and the potential for distributed networks of small radar systems," in Proceedings of the IEEE Radar Conference, pp. 1-3, IEEE, Pasadena, Calif, USA, May 2009.

[11] V. N. Bringi and V. Chandrasekar, Polarimetric Doppler Weather Radar; Principles and Attenuations, Cambridge University Press, Cambridge, UK, 2001.

[12] H. Godo, M. Naito, and S. Tsuchiya, "Improvement of the observation accuracy of X-band dual polarimetric radar by expansion of the condition to use KDP-R relationship," The Journal of Japan Society of Civil Engineers, Series B1, vol. 70, no. 4, pp. 505-510, 2014 (Japanese).

[13] MLIT, 2016, http://www.river.go.jp/kawabou/ipTopGaikyo.do.

[14] E. Nakakita, R. Nishiwaki, and K. Yamaguchi, "Detection of a system for early detection of baby rain cell aloft in a storm and risk projection for urban flash flood," Advances in River Engineering, vol. 20, 2014 (Japanese).

[15] R. Rotunno, "On the evolution of thunderstorm rotation," Monthly Weather Review, vol. 109, no. 3, pp. 577-586, 1981.

[16] J. B. Klemp, "Dynamics of tornadic thunderstorms," Annual Review of Fluid Mechanics, vol. 19, no. 1, pp. 369-402, 1987.

[17] W. R. Cotton, G. Bryan, and S. C. Van den Heever, Storm and Cloud Dynamics, vol. 99, Academic Press, 2010.

[18] E. Nakakita, R. Nishiwaki, H. Yamabe, and K. Yamaguchi, "Research on the prognostic risk of baby cell for guerilla-heavy rainfall considering by vorticity with doppler velocity," The Journal of Japan Society of Civil Engineers, vol. 69, no. 4, pp. 325330, 2013.

[19] A. Masuda and E. Nakakita, "Development of a technique to identify the stage of storm life cycle using X-band polarimetric radar," The Journal of Japan Society of Civil Engineers, Series B, vol. 70, no. 4, pp. 493-498, 2014 (Japanese).

[20] M. Katayama, A. Yamaji, F. Nakamura, H. Morita, and E. Nakakita, "Development of a system for early detection of a severe storm," Advances in River Engineering, vol. 21, 2015 (Japanese).
[21] M. Katayama, A. Yamaji, F. Nakamura, H. Morita, and E. Nakakita, "Development of detection system for a local rainstorm," in Proceedings of the River Engineering Symposium, 2015 (Japanese).

[22] E. Nakakita, H. Sato, and K. Yamaguchi, "Fundamental analysis of vortex tubes inside the baby cells of Guerrilla-heavy rainfall," The Journal of Japan Society of Civil Engineers, Series B, vol. 72, no. 4, 2016 (Japanese).

[23] E. A. Brandes, J. Vivekanandan, J. D. Tuttle, and C. J. Kessinger, "A study of thunderstorm microphysics with multiparameter radar and aircraft observations," Monthly Weather Review, vol. 123, no. 11, pp. 3129-3143, 1995.

[24] K. A. Scharfenberg, D. J. Miller, T. J. Schuur et al., "The joint polarization experiment: polarimetric radar in forecasting and warning decision making," Weather and Forecasting, vol. 20, no. 5, pp. 775-788, 2005.

[25] A. Adachi, T. Kobayashi, H. Yamauchi, and S. Onogi, "Detection of potentially hazardous convective clouds with a dualpolarized C-band radar," Atmospheric Measurement Techniques, vol. 6, no. 10, pp. 2741-2760, 2013.

[26] S. Shimizu and Y. Maesaka, "Multiple doppler radar analysis using variational technique to retriviete three-dimensional wind field," NIED Report, vol. 70, pp. 1-8, 2007 (Japanese).

[27] D. C. Liu and J. Nocedal, "On the limited memory BFGS method for large scale optimization," Mathematical Programming, vol. 45, no. 1, pp. 503-528, 1989. 

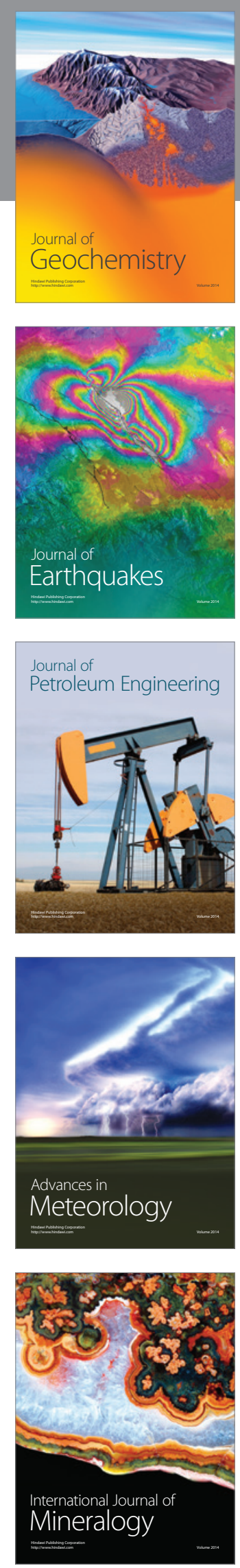
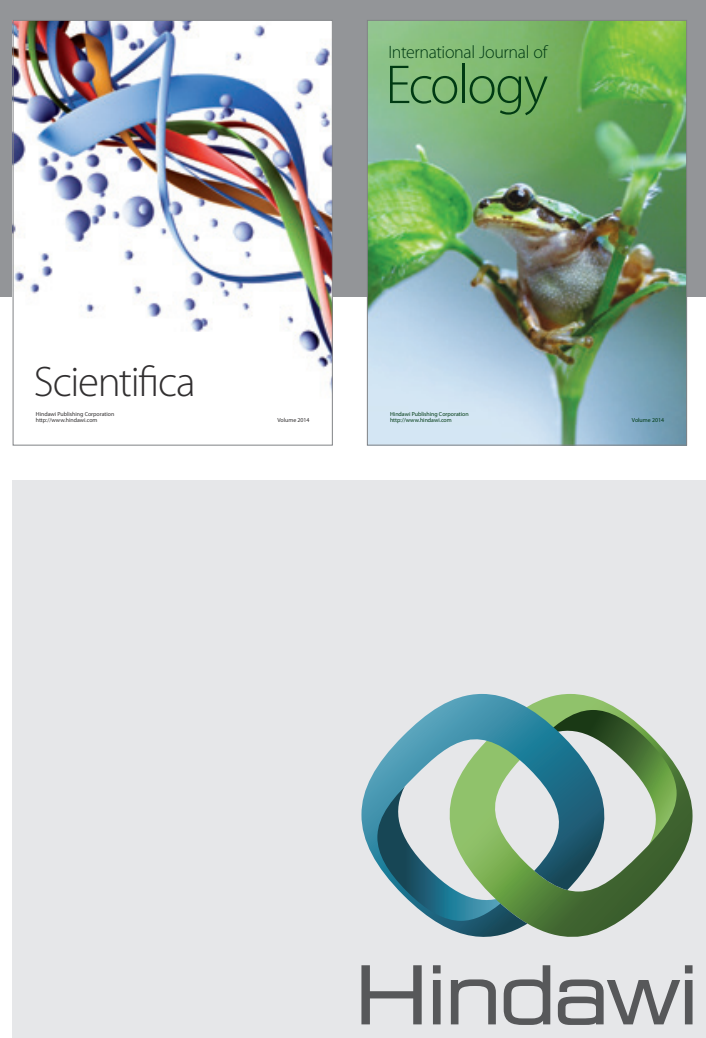

Submit your manuscripts at

https://www.hindawi.com
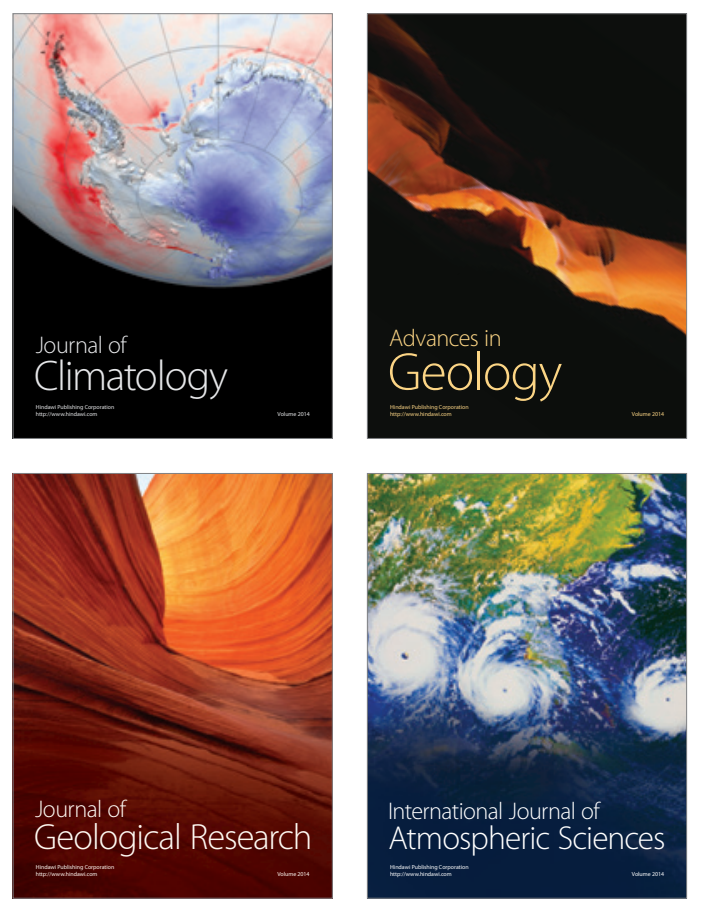

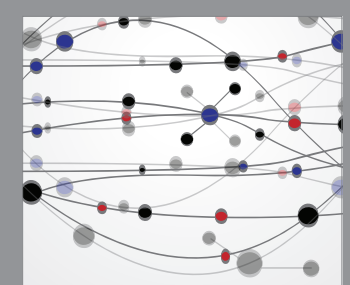

The Scientific

\section{World Journal}
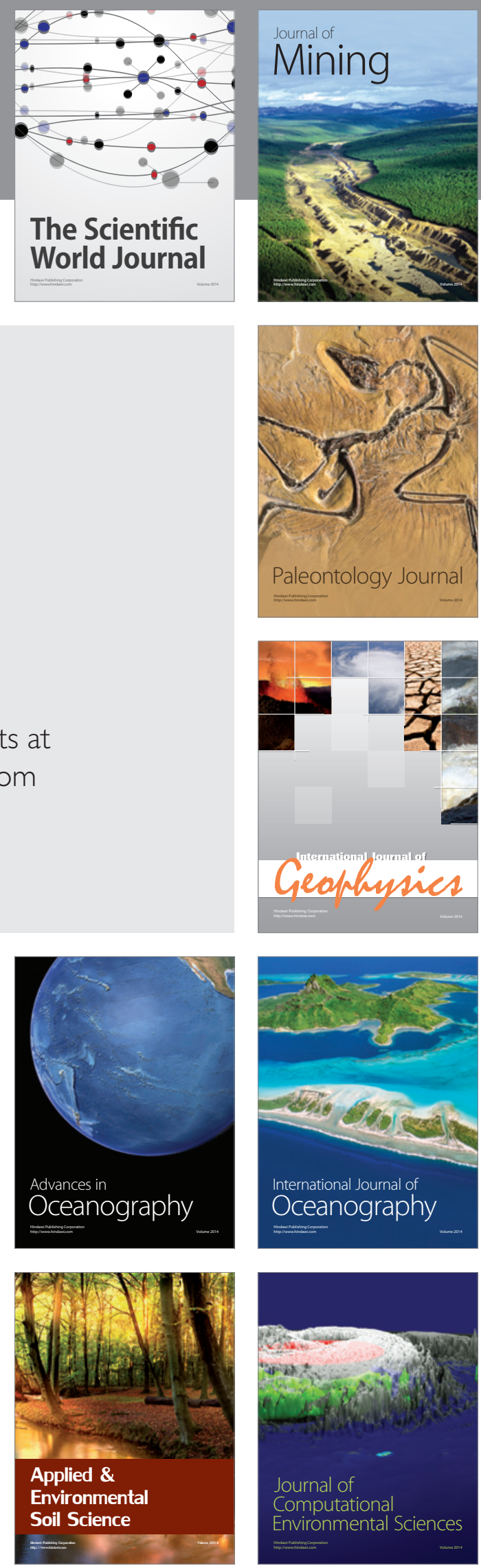